\title{
RESEARCH
}

Open Access

\section{Microbial community characterization of shrimp survivors to AHPND challenge test treated with an effective shrimp probiotic (Vibrio diabolicus)}

Leda Restrepo ${ }^{1,2,3}$, Cristóbal Domínguez-Borbor ${ }^{3}$, Leandro Bajaña ${ }^{3}$, Irma Betancourt ${ }^{3}$, Jenny Rodríguez ${ }^{3,4}$, Bonny Bayot ${ }^{3,5^{*}}$ and Alejandro Reyes ${ }^{1,2,6^{*}}$ (D)

\begin{abstract}
Background: Acute hepatopancreatic necrosis disease (AHPND) is an important shrimp bacterial disease caused by some Vibrio species. The severity of the impact of this disease on aquaculture worldwide has made it necessary to develop alternatives to prophylactic antibiotics use, such as the application of probiotics. To assess the potential to use probiotics in order to limit the detrimental effects of AHNPD, we evaluated the effect of the ILI strain, a Vibrio sp. bacterium and efficient shrimp probiotic, using metabarcoding (16S rRNA gene) on the gastrointestinal microbiota of shrimp after being challenged with AHPND-causing $V$. parahaemolyticus.

Results: We showed how the gastrointestinal microbiome of shrimp varied between healthy and infected organisms. Nevertheless, a challenge of working with AHPND-causing Vibrio pathogens and Vibrio-related bacteria as probiotics is the potential risk of the probiotic strain becoming pathogenic. Consequently, we evaluated whether ILI strain can acquire the plasmid pV-AHPND via horizontal transfer and further cause the disease in shrimp. Conjugation assays were performed resulting in a high frequency (70\%) of colonies harboring the pv-AHPND. However, no shrimp mortality was observed when transconjugant colonies of the ILI strain were used in a challenge test using healthy shrimp. We sequenced the genome of the ILI strain and performed comparative genomics analyses using AHPND and non-AHPND Vibrio isolates. Using available phylogenetic and phylogenomics analyses, we reclassified the ILI strain as Vibrio diabolicus. In summary, this work represents an effort to study the role that probiotics play in the normal gastrointestinal shrimp microbiome and in AHPND-infected shrimp, showing that the ILI probiotic was able to control pathogenic bacterial populations in the host's gastrointestinal tract and stimulate the shrimp's survival. The identification of probiotic bacterial species that are effective in the host's colonization is important to promote animal health and prevent disease.

(Continued on next page)
\end{abstract}

\footnotetext{
*Correspondence: bbayot@espol.edu.ec; a.reyes@uniandes.edu.co

${ }^{3}$ Escuela Superior Politécnica del Litoral, ESPOL, Centro Nacional de

Acuicultura e Investigaciones Marinas, CENAIM, Campus Gustavo Galindo Km

30.5 Vía Perimetral, P.O. Box 09-01-5863, Guayaquil, Ecuador

'Department of Biological Sciences, Universidad de los Andes, Bogotá,

Colombia

Full list of author information is available at the end of the article
}

(c) The Author(s). 2021 Open Access This article is licensed under a Creative Commons Attribution 4.0 International License, which permits use, sharing, adaptation, distribution and reproduction in any medium or format, as long as you give appropriate credit to the original author(s) and the source, provide a link to the Creative Commons licence, and indicate if changes were made. The images or other third party material in this article are included in the article's Creative Commons licence, unless indicated otherwise in a credit line to the material. If material is not included in the article's Creative Commons licence and your intended use is not permitted by statutory regulation or exceeds the permitted use, you will need to obtain permission directly from the copyright holder. To view a copy of this licence, visit http://creativecommons.org/licenses/by/4.0/ The Creative Commons Public Domain Dedication waiver (http://creativecommons.org/publicdomain/zero/1.0/) applies to the data made available in this article, unless otherwise stated in a credit line to the data. 


\begin{abstract}
(Continued from previous page)
Conclusions: This study describes probiotic bacteria capable of controlling pathogenic populations of bacteria in the shrimp gastrointestinal tract. Our work provides new insights into the complex dynamics between shrimp and the changes in the microbiota. It also addresses the practical application of probiotics to solve problems with pathogens that cause high mortality-rate in shrimp farming around the world.
\end{abstract}

Keywords: Acute hepatopancreatic necrosis disease (AHPND), Vibrio diabolicus, Probiotics, Metagenomics, Comparative genomics, Microbiome, Horizontal gene transfer (HGT)

\section{Introduction}

Bacterial pathogens cause considerable economic losses and constitute one of the biggest threats for sustainability of aquaculture worldwide $[1,2]$. Currently, one of the strongest emerging diseases for shrimp-producing countries is the acute hepatopancreatic necrosis disease (AHPND) [3]. The disease is caused by bacteria of the genus Vibrio harboring plasmids (pV-AHPND) that encode genes for the toxins PirA and PirB (Pir ${ }^{V P}$ ) [4]. AHPND causes high mortality through a severe peeling of the hepatopancreatic cells, leading to the shrimp's death $[5,6]$. This mortality leads to considerable economic losses in the shrimp farming industry. The extensive and excessive use of antibiotics has increased as a prophylactic treatment to control bacterial diseases in shrimp farms [7]. The systematic use of antibiotics is known to promote antibiotic resistance and remove beneficial microorganisms, which are essential for the healthy development of aquatic animals [7]. Consequently, there is a trend towards imposing more strict regulations on antibiotic use in aquaculture and to reduce the presence of antibiotic residues in aquaculture products [8]. Probiotics have thus emerged as a promising alternative to antibiotics and a way to treat and prevent cultured animal diseases [1].

Probiotics are microorganisms considered to provide beneficial effects to their hosts, either by preventing colonization of pathogenic bacteria through antagonism or promoting animal health through the stimulation of the immune system [1]. Current research in aquaculture focuses on establishing adequate standards on probiotics administration as well as providing an understanding on probiotics' effects on host-associated microbiota structure and health effects. For example, recent studies in humans demonstrated that the probiotic supplementation restored the normal microbiota composition and function in antibiotic-treated and cesarean-born infants [9]. Another study examined the effect on microbiome recovery after antibiotic therapy on humans, showing an increase in alpha diversity in the microbiomes of the group treated with probiotics [10]. There is thus a need to understand the roles of the microbiota in the health, growth, and survival of cultured aquaculture organisms. Studies in shrimp evaluating gastrointestinal microbial dynamics using probiotics are limited. One of the few studies available is that of Pinoargote et al. [11], where it was shown that probiotics had the ability to keep stable the microbiota in the shrimp's gastrointestinal tract, which could have positive effects on shrimp survival against AHPND. However, it is still needed to identify beneficial groups of microorganisms among the normal resident microbiota and the mechanisms underlying the interactions with probiotics in the gastrointestinal tract, in order to use them to help improve shrimp's health.

The choice of probiotics can be controversial in some cases, particularly in aquaculture, where certain probiotic genera are phylogenetically closely related to pathogens [12]. For example, in Ecuadorian shrimp hatcheries, beneficial strains belonging to the species Vibrio alginolyticus and AHPND-causing strains of the species Vibrio parahaemolyticus belong to the same phylogenetic clade, the Harveyi clade, and have been isolated from both, healthy and non-healthy larvae cultures [13]. In particular, a strain previously known as $V$. alginolyticus ILI strain was isolated from healthy shrimp larviculture [14]. Since then, it has been demonstrated to be an efficient probiotic agent through in vitro and in situ experiments [12, 14-16]. The Vibrio genus is a large and complex taxon that includes free-living bacteria of aquatic environments with the potential to reside in different animal hosts, including the gut and stomach of aquatic animals. The presence of related species with different adaptation strategies within a confined space under strong selection, such as different Vibrio species inside the gut of a crustacean, leads to the perfect setup for Horizontal Gene Transfer (HGT) events. This scenario can turn a previously commensal bacterium into a pathogen. We have recently shown the capacity of Vibrio to conjugate plasmids, even within distinct clades, and in consequence the transfer of pathogenic characteristics, including the infective capacity of AHPND [17]. pVAHPND has been identified not only among $V$. parahaemolyticus strains, but also in other related species: Vibrio harveyi, Vibrio owensii, and Vibrio campbellii $[5,16,18$ 20], within the Harveyi clade, and Vibrio punensis of the Orientalis clade [17].

Although the potential HGT of pathogenicity traits coded in plasmids may be essential to transfer 
pathogenic properties to genetically related bacteria, AHPND progression does not depend solely on the presence of toxins encoded by the plasmid pV-AHPND. Li et al. [18] studied the repertoire of associated virulence factors in AHPND-causing $V$. parahaemolyticus by means of a comparative genomic analysis using the clinical strain RIMD22106 and both, environmental strains not producing AHPND, as well as toxic isolates producing AHPND. The authors described two secretion systems (type III and VI) present in two copies in the pathogenic strains. Specifically, T3SS1 is present in all sequenced strains of $V$. parahaemolyticus. It contains 49 genes located on chromosome I [18], including one putative effector protein VstL (homologous to SctL) that is critical for injectosome recruitment and could help with rapid cell death against multiple eukaryotic cell lines [18]. In addition, T3SS2 is composed of 12 genes, two of which (trh and $t d h$ ) share $70 \%$ identity with each other and are also present in the human pathogenic strains in $V$. parahaemolyticus. TDH is a pore-forming toxin and TRH is a thermolabile toxin and immunologically similar to TDH [19]. All AHPND-causing strains, but none of the non-AHPND strains, have been described to harbor the antibacterial type VI secretion system 1 (T6SS1), which was previously identified and characterized in the clinical isolate RIMD2210633 and is characterized by containing 42 genes [18]. This finding suggests a fitness advantage provided by the T6SS1 acquisition of AHPN D-causing $V$. parahaemolyticus over competing bacteria, which could facilitate shrimp infection. Thus, the diversity of AHPND-causing pathogens and the different pathogenicity factors they may harbor raises the question of whether other Vibrio-related bacteria commonly used as probiotics, such as the ILI strain, could be a safe probiotic or whether or not it could potentially turn into a pathogenic strain.

We aimed to assess the response of the Shrimp's microbiome to a challenge with AHPND-causing bacteria and test whether the presence of a probiotic, the genetically related ILI strain, could modify such response. Furthermore, we wanted to evaluate if the phylogenetically related ILI strain was capable of acquiring the plasmid pV-AHPND and cause the disease in shrimp. To this end, we performed conjugation assays using a $V$. parahaemolyticus strain harboring the $\mathrm{pV}$ AHPND plasmid as a donor and the ILI strain as a potential recipient. Challenge tests were performed on the Penaeus vannamei shrimp. Finally, we aimed to better understand the genomic and ecological characteristics of the ILI probiotic and their implications on the interaction with AHPND-causing V. parahaemolyticus. Thus, we sequenced its genome and performed a comparative genomics analysis with a selected set of AHPND and non-AHPND causing $V$. parahaemolyticus isolates, as well as non-AHPND ILI strain-related isolates. Our findings suggest that the ILI strain is a safe probiotic and can be used in AHPND conditions due to the absence of pathogenicity, even after the acquisition of the $\mathrm{pV}$ AHPND plasmids.

\section{Materials and methods Ethics statement}

All procedures were approved by the Ethics and Animal Welfare Committee of ESPOL and CENAIM.

\section{Growth condition of the probiotic strains}

We used the ILI probiotic strain provided by the Microbiology Laboratory at the Centro Nacional de Acuicultura e Investigaciones Marinas (CENAIM), previously identified as $V$. alginolyticus [13] and characterized as an efficient probiotic [12-16]. This strain was originally isolated from water of a healthy larvae hatchery and has been part of the CENAIM culture collection for over 26 years. The ILI strain was aerobically grown in TSA (Tryptic Soy Agar) with $2 \% \mathrm{NaCl}$ by seeding and incubated for $24 \mathrm{~h}$ at $28^{\circ} \mathrm{C}$. Single colonies were selected and transferred to new TSA plates with $2 \% \mathrm{NaCl}$ for the conjugation assays [20].

The Bacillus sp. strain P64 was originally isolated from wild adult shrimp and has been part of the culture collection at CENAIM since 2004. This strain has been reported to have positive probiotic effects [13-16]. Growth of this strain was performed on marine agar with $2 \%$ $\mathrm{NaCl}$ as described previously [15].

\section{Preparation of probiotics inoculum}

To generate the inoculum, bacterial probiotic strains were streaked in LB agar with $2 \% \mathrm{NaCl}$ and incubated for $18 \mathrm{~h}$. One colony for each strain was used to inoculate $10 \mathrm{~mL}$ of $\mathrm{LB}$ broth. Successive inoculations were made to scale up the volume. Absorbance was adjusted to optical density (OD) at $540 \mathrm{~nm}$, approximately $2 \times$ $10^{5}$ CFUs (ILI strain) and $2 \times 10^{8}$ CFUs (Bacillus strain). Each bacterium was added by spraying daily in the commercial food ( $35 \%$ protein) and immediately administered with the feeding to the shrimp.

\section{Immersion challenge test of shrimp supplemented with probiotics}

Shrimp were supplemented for a 1-month period with the ILI and the Bacillus probiotic strains and then challenged with the AHPND-causing $V$. parahaemolyticus BA94C2 strain to further evaluate its effects on the microbial community. The BA94C2 strain was isolated from diseased organisms in South America and belongs to the Centro Nacional de Acuicultura e Investigaciones Marinas culture collection since 2015. Four treatments were evaluated. Shrimp that did not receive probiotic 
and were challenged only with media without bacterium (healthy controls; $\mathrm{C} 0$ ), shrimp that did not receive probiotics but were challenged (C1), shrimp that received the ILI probiotic and were challenged (C2), and shrimp that received the Bacillus sp. strain P64 probiotic and were challenged as well (C3). The latter was included to compare its mortality with the ILI probiotic at the end of the challenge test with AHPND. Probiotics were administered for 1 month using the treated feed twice a day (water salinity $\sim 34 \mathrm{gl}^{-1}$ and temperature $\sim 28^{\circ} \mathrm{C}$ ). The commercial feed dose used was $3 \%$ of the average body weight of the shrimp (adjusted according to the food consumed). Environmental parameters such as salinity, temperature, and dissolved $\mathrm{O}_{2}$ and were monitored daily in ponds and tanks after the challenge. Water samples from each treatment were collected before adding probiotics from each tank to evaluate the presence of the probiotic in the tank.

After feeding the shrimp with the corresponding probiotic treatments, survival of the animals to a challenge with $V$. parahaemolyticus BA94C2 strain was evaluated. Completely randomized design was applied with four treatments and six replicates per treatment. Experimental units ( $40 \mathrm{~L}$ tanks) were stocked with $40 \mathrm{P}$. vannamei shrimp (average weight $=2.5 \pm 0.3 \mathrm{~g}$ ). During the assay, shrimp were monitored for mortality every $2 \mathrm{~h}$. Difference of cumulative mortalities at $50 \mathrm{~h}$ post infection among infected treatments were analyzed with the Kruskal-Wallis test using the R software [21]. Shrimp survival was recorded at the end of the experiment. The water exchange $(50 \%)$ was performed daily before and after the challenge was performed, including during the period of probiotic feeding, but not the $50 \mathrm{~h}$ challenge period. Shrimps were exposed to a $12 \mathrm{~h}$ photoperiod per day. Pairwise comparisons using Nemenyi test were conducted to evaluate the status (healthy, diseased and with probiotics) to bacterial community variation using $R$ software. Mortalities were expressed as mean \pm standard error.

\section{Re-isolation of bacteria}

Shrimp from all treatments were collected at $12 \mathrm{~h}$ post infection. Hepatopancreas and stomachs were removed from shrimp and macerated in sterile saline solution. Serial dilutions of $100 \mu \mathrm{L}\left(10^{-1}, 10^{-2}\right.$ and $\left.10^{-3}\right)$ containing macerated hepatopancreas and stomachs were inoculated on TCBS agar and incubated at $28^{\circ} \mathrm{C}$ for $18 \mathrm{~h}$, and the three most predominant colonies were purified on TSA plates. Afterwards, the DNA of the colonies was extracted and stored for subsequent comparisons with the tested isolates using Pir $^{V P}$ gene detection (Table S1), as described below. Macerates of each shrimp hepatopancreas and stomachs of healthy controls ( $\mathrm{C} 0)$ were also analyzed for $\mathrm{Pir}^{V P}$ gene detection.

\section{Metagenomics sample collection}

Moribund shrimp exhibited the usual clinical and pathological symptoms of the AHPND, such as lethargy, empty intestine, and pale hepatopancreas. Stomachs and hepatopancreas from each shrimp were aseptically dissected. The tissues were preserved individually in RNAlater solution and stored at $-80^{\circ} \mathrm{C}$ until subsequent DNA extraction. After performing the sequencing quality evaluation and trimming (see below), a total of 41 samples of $P$. vannamei shrimp were retained-18 from the healthy shrimp treatment (nine hepatopancreas and nine stomachs) and 13 AHPND-infected shrimp not treated with probiotics (eight stomachs and five hepatopancreas) - and five samples from each of the probiotictreated groups -4 hepatopancreas and 1 pool of 5 stomachs. The stomach tissue of the surviving shrimp was pooled due to the low biomass recovered.

\section{S rRNA gene sequencing}

DNA was extracted from stomachs and hepatopancreas using the PureLink Genomic DNA Mini Kit (Invitrogen; catalog no. K1820-00) according to the manufacturer instructions. DNA was quantified using a Qubit fluorometer HS assay Kit (Life Technologies). The hypervariable $\mathrm{V} 4$ region of the $16 \mathrm{~S}$ rRNA gene was sequenced using the $515 \mathrm{~F} / 806 \mathrm{R}$ PCR primers [22]. Indexed amplicons were pooled and sequenced on the Illumina MiSeq platform, with $2 \times 250 \mathrm{bp}$ paired-end reads. A total of 48 samples were sequenced at Macrogen by using MiSeq 2000 (Illumina, San Diego). Data generated has been deposited in GenBank under BioProject accession number PRJNA580262.

\section{Data pre-processing and ASV picking}

Amplicon sequence variants (ASV) were analyzed with QIIME2 version 2019.1 [23]. Reads were imported into QIIME2 and, subsequently, forward and reverse amplicon sequences were merged with an overlap of $200 \mathrm{bp}$ and demultiplexed according to sample specific indexes, and redundant sequences were removed. Sequences were filtered and denoised into features with DADA2 [24]. In order to obtain a comprehensive description of the within-sample bacterial community, alpha diversity metrics (species richness, Chao1, Shannon diversity and total number of observed OTUs) and rarefaction plots with 3000 sequences per sample were generated. Pairwise comparisons using Wilcoxon rank sum tests were conducted to quantitatively evaluate ASV variations between treatments of healthy, diseased and treated with probiotics, using $\mathrm{R}$ software [21]. Likewise, multivariate analyses of beta diversity (Bray-Curtis, Weighted and Unweighted UniFrac) were conducted to assess differences in bacterial community compositions between samples from (a) healthy shrimp (stomach and 
hepatopancreas), (b) healthy and AHPND-infected shrimp ( $\mathrm{C} 0$ and $\mathrm{C} 1$ ), and (c) shrimp treated with the two probiotic treatments ( $\mathrm{C} 2$ and $\mathrm{C} 3)$. In all cases, we considered taxa accounting for a cumulative $96 \%$ of total abundance. In order to visualize these differences, we performed principal coordinate analyses (PCoA) calculating the Bray-Curtis dissimilarity (Beta diversity). Taxonomic assignments of representative sequences were conducted using the Silva database as reference to classify $16 S$ rRNA gene OTUs clustered at 99\% similarity [25]. Differential abundance of taxa was determined based on changes to the Shannon diversity estimates. To determine taxa significantly enriched in healthy shrimp and shrimp treated with probiotics, we applied a linear discriminant (LDA) effect size (LEfSe) analysis method [26].

\section{Conjugation assays and evaluation of the pathogenicity of the ILI transconjugated bacterial strain}

The ILI strain was used as the recipient strain in conjugation experiments. The $V$. parahaemolyticus BA94C2 strain harboring the plasmid pV-AHPND [27] was used as donor. To evaluate the capacity of conjugation, experiments were designed following the protocol suggested by Llosa et al. [20] under controlled laboratory conditions simulating natural microenvironments. Control mattings in nutrient TSB broth were performed avoiding contact between strains. Both donor and recipient strains were incubated overnight in LB agar with $2 \%$ $\mathrm{NaCl}$ at $42{ }^{\circ} \mathrm{C}$ and diluted in $\mathrm{NaCl}$ to approximately $10^{8}$ cells per $\mathrm{mL}$ by using McFarland's nephelometer standard. One milliliter of broth containing the donor strain was mixed with $1 \mathrm{~mL}$ of broth containing the recipient strain, plus $2 \mathrm{~mL}$ of TSB (tryptic soy broth), and incubated for $24 \mathrm{~h}$ at $28^{\circ} \mathrm{C}$. After incubation, the conjugation mixtures were diluted 10 -fold in $0.9 \%$ saline solution to a $10^{-7}$ dilution. For each sample, $0.1 \mathrm{~mL}$ of the dilution was spread on a TSA agar plate with $2 \% \mathrm{NaCl}$. The colonies were differentiated by the clear morphological characteristics of the ILI colonies (smaller, with growth following a filamentous shape) and BA94C2 donor (convex, entire and well-defined colonies in appearance, with a size of 7-10 $\mathrm{mm}$ in diameter). Colonies were incubated at $28^{\circ} \mathrm{C}$, and then counted and replicated. The presence of the pV-AHPND plasmid and, hence, the detection of transconjugants was performed by PCR amplification of the PirA and PirB plasmid genes [27] as explained below.

The pathogenicity of the ILI transconjugant bacterial strain was evaluated by an immersion challenge test. Briefly, a single colony of the ILI transconjugant strain was picked and re-suspended in $30 \mathrm{~mL}$ of sterile TSB. The culture was incubated for $18 \mathrm{~h}$ at $28^{\circ} \mathrm{C}$ on a rotary shaker $(200 \mathrm{rpm})$, and the bacterial density was determined on a microplate reader (Varioskan ${ }^{\mathrm{Tm}}$, Thermo Fisher Scientific). One $\mathrm{mL}$ of this solution was transferred to $50 \mathrm{~mL}$ of TSB medium and cultured as described below for $10 \mathrm{~h}$. The suspension was plated onto TSA plates after serial dilutions to determine the colony-forming units (CFU) of the isolate. The $V$. parahaemolyticus BA94C2 [28] strain was used as positive control under the same conditions. Shrimp were obtained from a pond without the symptomatology of AHPND disease, and the challenge was performed as described by Tran et al. [5], with minor modifications as follows. For the challenge test, transconjugant strains were cultured in TSB for $12 \mathrm{~h}$ at $28^{\circ} \mathrm{C}$, until bacterial density reached $2 \times 10^{9} \mathrm{CFU} \mathrm{mL}^{-1}$. Then, a $15-\mathrm{min}$ immersion was performed on tanks containing 40 healthy shrimp $(2.5 \pm 0.5 \mathrm{~g})$ per flask and a solution of bacterial suspension with sterile saline water, to achieve a bacterial density of approximately $2 \times 10^{8} \mathrm{CFU} \mathrm{mL} \mathrm{m}^{-1}$. Immersed animals were transferred into experimental aquariums filled with $40 \mathrm{~L}$ of filtered and UV sterilized seawater and continuously aerated. After transfer, a solution of bacterial suspension was immediately added directly to the experimental tanks containing clean seawater (filtered and UV sterilized), to reach an approximate bacterial density of $2 \times 10^{6} \mathrm{CFU} \mathrm{mL} \mathrm{m}^{-1}$. A negative control which consisted of 40 shrimp immersed in sterile TSB, without bacterial strains, was also included. All treatments, including the control, had 10 replicates. During the assay, shrimp were monitored for mortality observations every $2 \mathrm{~h}$. Difference of cumulative mortality at $50 \mathrm{~h}$ post infection among infected treatments was analyzed with Wilcoxon's rank sum test by using the $\mathrm{R}$ software [29]. Mortalities were expressed as mean \pm standard error.

\section{Bacterial culture DNA extraction}

DNA was extracted with the PureLink Genomic DNA Mini Kit (Invitrogen; catalog no. K1820-00) according to the manufacturer's instructions with the following modifications: (a) $1 \mathrm{~mL}$ of a bacterial culture grown overnight was centrifuged at $4000 \mathrm{rpm}$ for $10 \mathrm{~min}$. The supernatant was removed, and $1 \mathrm{~mL}$ of digestion buffer was added. (b) PureLink Genomic Digestion Buffer from the PureLink Genomic DNA Mini Kit and proteinase K (final concentration $2 \mathrm{mg} / \mathrm{ml}$ ) was added and incubated at $55^{\circ} \mathrm{C}$ for $2 \mathrm{~h}$, followed by centrifugation at $5000 \mathrm{rpm}$ for 2 min. (c) After the DNA was cooled at room temperature, it was extracted using the PureLink Genomic DNA Mini Kit (Invitrogen; catalog no. K1821-04) based on binding of the DNA to silica columns, in accordance with the manufacturer's instructions. (d) The supernatant was discarded, and the samples were air dried for $3 \mathrm{~h}$. DNA extracts were stored at $-20^{\circ} \mathrm{C}$ for further analysis. DNA concentrations were determined 
using a NanoDrop 8000 Spectrophotometer (NanoDrop 2000, Thermo Fisher Scientific Inc.).

\section{$\operatorname{Pir}^{V P}$ gene detection}

The presence of $\mathrm{Pir}^{V P}$ was tested on 152 colonies of donors, recipients, and isolated bacterial strains of transconjugants using a nested PCR method, with primers previously described to obtain amplified products of 1269 and $230 \mathrm{bp}$ (Table S1). Likewise, PirA and PirB genes were amplified separately with specific primers aimed at generating PCR products of 284 and $392 \mathrm{bp}$ for PirA and PirB, respectively (Table S1). PCR was carried out in a $10 \mu \mathrm{l}$ reaction volume containing $10 \mathrm{mM}$ Tris$\mathrm{HCl}, \mathrm{pH} 8.3,50 \mathrm{mM} \mathrm{KCl}, 3 \mathrm{mM} \mathrm{MgCl} 2,100 \mathrm{mM}$ of each $\mathrm{dNTP}, 0.4 \mathrm{mM}$ of the specific primer, $2 \mu \mathrm{l}$ of genomic DNA, and $1 \mathrm{U} / \mu \mathrm{l}$ Taq DNA polymerase (Invitrogen). Amplifications were performed using a Thermo Fisher thermoblock under the following conditions: $4 \mathrm{~min}$ initial denaturation at $94{ }^{\circ} \mathrm{C}$, followed by 39 cycles of $5 \mathrm{~s} \mathrm{de}-$ naturation at $94{ }^{\circ} \mathrm{C}, 45 \mathrm{~s}$ annealing at $46^{\circ} \mathrm{C}$, and $1 \mathrm{~min}$ elongation at $72{ }^{\circ} \mathrm{C}$, with a final elongation step at $72{ }^{\circ} \mathrm{C}$ for $5 \mathrm{~min}$ (13). The PCR products $(5 \mu \mathrm{l})$ were analyzed on a 1.5\% agarose gel stained with SYBR Safe DNA Gel Stain (Thermo Fisher), visualized under UV transillumination and photographed using an E-Gel Imager (Thermo Fisher).

\section{Genome sequencing and de novo assembly}

To sequence the genome of the ILI strain, genomic DNA was prepared according to a guide for preparing SMRTbell template for sequencing on the PacBio RS System developed by Pacific Biosciences (catalog number: 100-938-900). The template was sequenced in Macrogen Korea using SMRT Sequencing. SMRTbell ${ }^{\mathrm{TM}}$ hairpin loop adapters were ligated to both ends of the double stranded DNA insert to produce a SMRTbell ${ }^{\mathrm{TM}}$ sequencing template. Adapter sequences were removed from a CCS (or consensus circular sequencing) read and the read was partitioned to form one or more subreads, which contained the sequence from a single pass of a polymerase on a single strand of an insert within a SMRTbell ${ }^{\mathrm{TM}}$. The subreads contained the full set of quality values and kinetic measurements. Reads were trimmed to include only the high-quality regions. Reads containing ambiguous base calls or barcode errors were discarded. De novo assembly was performed using Canu [30]. The complete genome sequence was deposited at DDBJ/EMBL/GenBank under the BioProject accession number PRJNA580299.

\section{Genome annotation}

The genome of the ILI strain was annotated using Prokka and RAST [31, 32]. All predicted ORFs were translated and searched (BLAST) against (1) non- redundant protein databases from NCBI (E-value threshold $<10^{-6}$ and minimal alignment length $\geq 80 \%$ ), (2) Swiss-Prot, (3) Clusters of Orthologous Groups (COG), (4) Kyoto Encyclopedia of Genes and Genomes (KEGG), and (5) Gene Ontology (GO) [33-35].

\section{Multilocus sequence typing phylogeny}

A multilocus sequence typing (MLST) analysis was performed with the phylogenetic marker genes: fts $Z$, gapA, gyrB, mreB, topA, and $16 S$ rRNA, from a dataset of 78 related Vibrio species obtained from the NCBI Reference Sequence Database (RefSeq). GenBank accession numbers are listed in Table S2. We used the TCS program [36] to calculate a phylogenetic network estimation among Vibrio species used for the MLST analysis and to choose the most related strain to the ILI strain. Sequences were aligned with MEGA 6.0 [37] using the MUSCLE algorithm. Phylogenetic trees were constructed using maximum likelihood (ML). We use JModeltest 2.0 [38] to test the evolution models based on the hierarchical likelihood ratio test, determining that the $\mathrm{TIM}+\mathrm{G}$ model best fit the data.

\section{Comparative genome analysis and genomic island identification}

MAUVE software [39] was used to perform whole genome alignments and comparisons. The genome of the ILI strain was aligned to the two most cured, wellannotated, and complete genomes of the related species (V. alginolyticus strain NBRC $15630=$ ATCC 17749 and $V$. antiquarius strain EX25, accession numbers: GCA 000354175.2 and GCA_001909305.1, respectively) to evaluate gene conservation. Genes previously predicted using Pfam for the identification of genomic islands (GIs) and several common characteristics of the genome, such as abnormal sequence composition and the presence of mobile genetic elements were used [40].

\section{Average nucleotide identity, correlation indexes and DDH estimates}

Full genome comparison statistics were determined with the Genome-to-Genome Distance Calculator (GGDC 2.1, http://ggdc.dsmz.de/distcalc2.php). The average nucleotide identity (ANI) was measured between pairs of genomes, based on the BLAST algorithm (ANIb). In addition, we calculated the tetranucleotide frequencies (TETRA) for the different genomes. The ANIb and TETRA values of the Vibrio genome comparisons were calculated for all possible bacterial pairs, including all species identified in the GenBank database as $V$. antiquarius (three species), 16 species of $V$. alginolyticus, and the ILI strain. All comparisons were represented as heatmaps using the R statistical software [21]. 


\section{Results}

Probiotics, in particular ILI strain prevents dysbiosis of the microbiota caused by AHPND

We evaluated the changes of the microbial community structure caused by AHPND on shrimp stomachs and hepatopancreas, with and without the presence of probiotics. Shrimp were either not challenged (healthy controls; C0) or challenged with AHPND causing $V$. parahaemolyticus BA94C2 strain (AHPND-infected controls; C1) and compared with the same setup but with the daily supplementation of a probiotic (via feeding in the commercial food without other probiotic medication) for a 1-month period prior to the challenge. We used as probiotic the ILI strain (C2) and another probiotic (Bacillus sp. strain P64) for comparison (C3). The results from the challenge test showed the highest survival values for treatments $\mathrm{C} 0$ and $\mathrm{C} 2(P<0.001$, for both treatments in comparison with the $\mathrm{C} 1$ and $\mathrm{C} 3$ treatments, Nemenyi test), followed by $\mathrm{C} 3$, and finally shrimp that showed the highest mortality were those of the AHPND-infected shrimp treatment (C1) (Fig. S1).

Characterization of the microbiota of stomachs and hepatopancreas through V4 $16 S$ rRNA gene sequencing was performed using an Illumina MiSeq v3 kit $(2 \times 250$ $\mathrm{PE})$. A total of $58,074,501$ high-quality reads were obtained from 42 samples (3000-49,057 reads per sample). Rarefaction curves showed sampling saturation was achieved well before 3000 reads (Fig. S2). In order to remove the minimum number of samples, a rarefaction of the data to 3000 sequences per sample was performed, resulting in a total of 737 ASVs across samples. The final dataset contained information for nine hepatopancreas and nine stomachs of healthy shrimp, five hepatopancreas and eight stomachs of AHPND-infected shrimp, and four hepatopancreas and one pool of five stomachs for each one of the probiotic treatments.

The diversity of the microbiota in healthy shrimp (C0) was significantly higher than on AHPND-infected shrimp (C1), as observed using different alpha diversity metrics (Fig. 1). In particular, Shannon diversity showed a high diversity in shrimp treated with the ILI probiotic (C2), equivalent to that of healthy shrimp (C0) (healthy $4.24 \pm 0.89$ vs. infected $3.42 \pm 0.90 ; P=0.861$, Nemenyi test) in accordance to the mortality rates displayed. Equivalent results were obtained with Chao1 (healthy $934 \pm 8.21$ vs. infected $822 \pm 3.41 ; P=0.786$, Nemenyi test). Furthermore, although the Bacillus probiotic (C3) had lower diversity than infected controls (C1), no statistical significance was observed $(2.45 \pm 0.41, P=0.149$, Nemenyi test).

Upon analysis of the microbial community structure, Proteobacteria, Firmicutes, and Tenericutes were the most abundant phyla, i.e., their accumulated abundance is higher than $90 \%$ across treatments, both in hepatopancreas (hp) and stomach samples (st) (Table 1). A reduction in the number of phyla was observed in

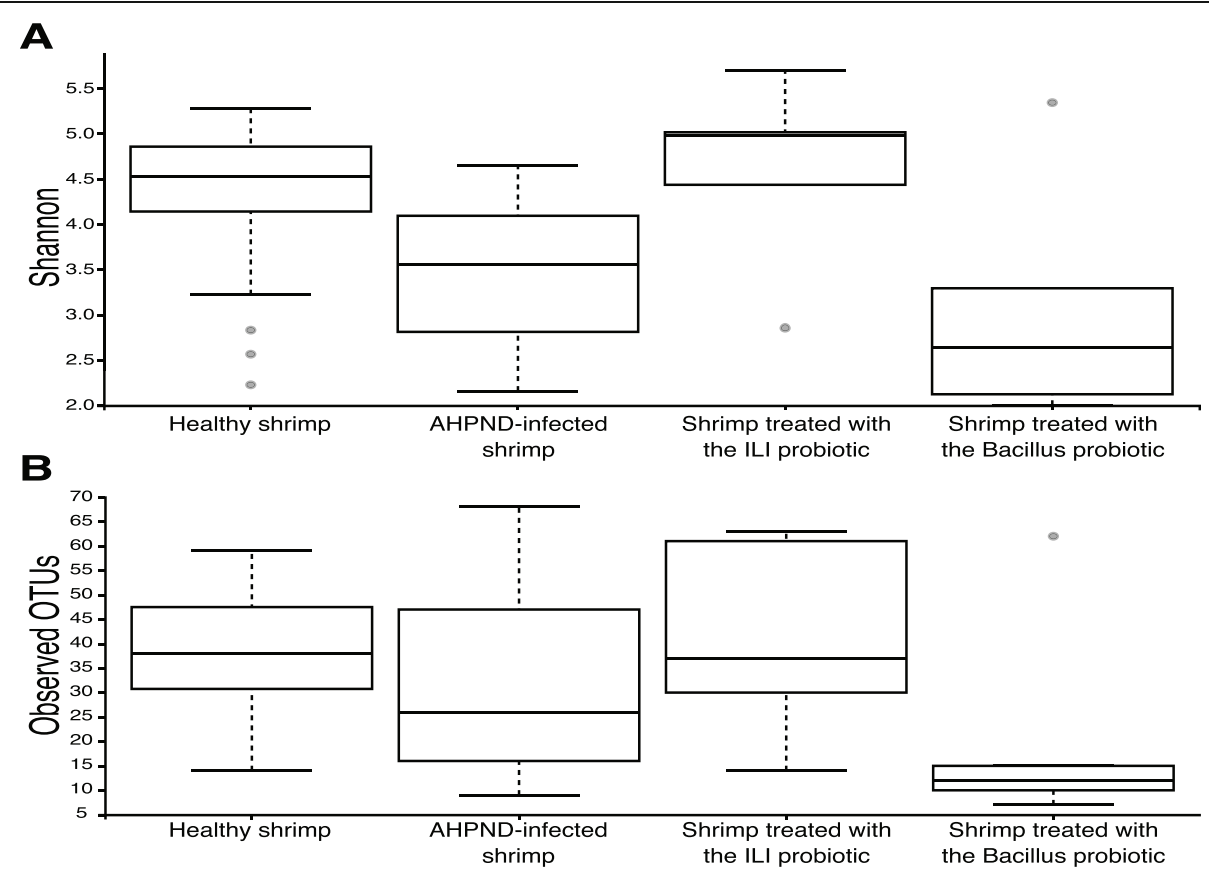

Fig. 1 Microbial alpha diversity is reduced by AHPND. Boxplots of two different alpha diversity indexes are shown for all samples grouped by health status a Shannon index and $\mathbf{b}$ number of observed OTUs (ASVs). More variation in the composition of gastrointestinal microbiota for healthy shrimp samples is observed. An increase was observed in the indexes for shrimp treated with the ILI probiotic. There is a stronger effect on diversity (Shannon) than on richness (Observed OTUs) by the treatments 
hepatopancreas and stomachs of AHPND-infected shrimp (hp $=12$ phyla and st $=4$ phyla) compared with healthy animals $(\mathrm{hp}=14$ phyla and st $=8$ phyla), except for shrimp treated with the ILI probiotic, supporting the previous observation regarding alpha diversity. Even though Proteobacteria was the most abundant phyla overall, the rank abundance of the next most abundant phyla varied among different treatments and organs. For example, hepatopancreas from shrimp treated with ILI resemble most closely resemble the rank abundance of healthy shrimp (Table 1, Fig. 2a).

At the family level, the most abundant taxa across all samples are shown in Table 2. The most abundant family could be assigned taxonomically at the order level (Vibrionales). Similar to the observation at the phylum level, challenged shrimp showed a reduction in diversity by expansion of the Vibrionales. This was observed in both tissues and treatments, except for ILI treated animals (Fig. 2b). Interestingly, ASVs assigned at family level to the Vibrionales or Vibrionaceae were expanded in particular in the stomach of infected individuals, thus showing that more than one Vibrionales species were increasing their abundance in affected shrimp.

To compare the community structure among treatments, a beta diversity analysis, using the Bray-Curtis dissimilarity metric, was performed. Comparisons between healthy (C0) and AHPND-infected shrimp (C1) showed that there was no clear clustering between treatments in hepatopancreas samples (Fig. 3a). Conversely, stomach samples clustered depending on the health status of shrimp (Fig. 3b), indicating that AHPND infection clearly affects the configuration of the gastrointestinal microbiota. Even though hepatopancreas samples treated with the probiotic did not form a clear cluster, these $(\mathrm{C} 1)$ showed a reduced distance to the healthy shrimp (CO) compared to the infected animals. In pooled stomach samples, those treated with probiotics had intermediate positions between healthy and infected samples, but ILI-treated samples (C2) grouped closer together with controls $(\mathrm{C} 0)$, while samples treated with Bacillus probiotics clustered together with the infected ones.
A linear discriminant analysis effect size (LEfSe) was conducted to determine the significant differences in the abundance of ASVs between healthy and AHPND-infected shrimp. This analysis was calculated for each ASV identified in healthy and infected samples and was used to determine potential biomarkers in each microbiome of the probiotics tested. In hepatopancreas, changes in the relative abundance of seven ASVs were the main contributors to the observed diversity differences, through enrichment on infected individuals (Fig. 4a). It is noteworthy that all seven ASVs were assigned to the Phylum Proteobacteria, and three of these were assigned to the order Vibrionales, but no further taxonomical assignment was possible.

In stomach samples, fourteen ASVs showed differential abundance among treatments. Four ASVs were significantly over abundant in AHPND-infected shrimp (Vibrionales 1, Vibrionales 3, Vibrionales 2, and Vibrionales 4), whereas the remaining ten ASVs were significantly more abundant in healthy shrimp (Fig. 4b). Analogous to what was found in hepatopancreas, only members of the Proteobacteria, and in this particular case of the Vibrionales, were among the disease-related taxa. Taxa enriched in control samples included members of the Tenericutes (4), Proteobacteria (4), and Bacteroidetes (2), reflecting a broader taxonomical diversity in healthy microbiota. Within the 4 Proteobacteria taxa increased in control samples, only one member of the Vibrionaceae family (Photobacterium damselae) was found (Table 3), but its potential role in the shrimp's health is not clear.

Given that taxonomic assignments of ASVs among Vibrionales reached the family rank at best, we evaluated nucleotide dissimilarity among these ASVs in order to identify species- or clade-specific SNPs that will allow a further taxonomic assignment. Specifically, we aimed to determine whether some of these ASVs corresponded to the genus Vibrio, which are of great importance in the cultured shrimp microbiota. We identified SNPs that allowed the clustering of related variants. However, the SNPs themselves did not provide enough resolution to resolve at the species level, but the clustering allowed to

Table 1 Values (percent) of relative abundance of the four most abundant phyla for healthy shrimp, AHPND-infected shrimp, and shrimp treated with ILI or the Bacillus probiotic

\begin{tabular}{|c|c|c|c|c|c|c|c|c|}
\hline \multicolumn{9}{|l|}{ Phyla level } \\
\hline & \multicolumn{2}{|c|}{ Healthy } & \multicolumn{2}{|c|}{ AHPND-infected } & \multicolumn{2}{|c|}{ Treated with the ILI probiotic } & \multicolumn{2}{|c|}{ Treated with the Bacillus probiotic } \\
\hline & $\mathrm{Hp}$ & St & $\mathrm{Hp}$ & St & $\mathrm{Hp}$ & St & $\mathrm{Hp}$ & St \\
\hline Proteobacteria & 77.95 & 89.81 & 94.45 & 99.84 & 79.33 & 94.93 & 99.08 & 98.55 \\
\hline Firmicutes & 9.38 & 1.46 & 3.33 & 0 & 7.56 & 0 & 0.33 & 0 \\
\hline Tenericutes & 6.89 & 6.65 & 0 & 0.02 & 10.39 & 4.92 & 0 & 0 \\
\hline Cyanobacteria & 4.74 & 0.73 & 1.59 & 0.06 & 2.03 & 0 & 0.65 & 1.12 \\
\hline
\end{tabular}




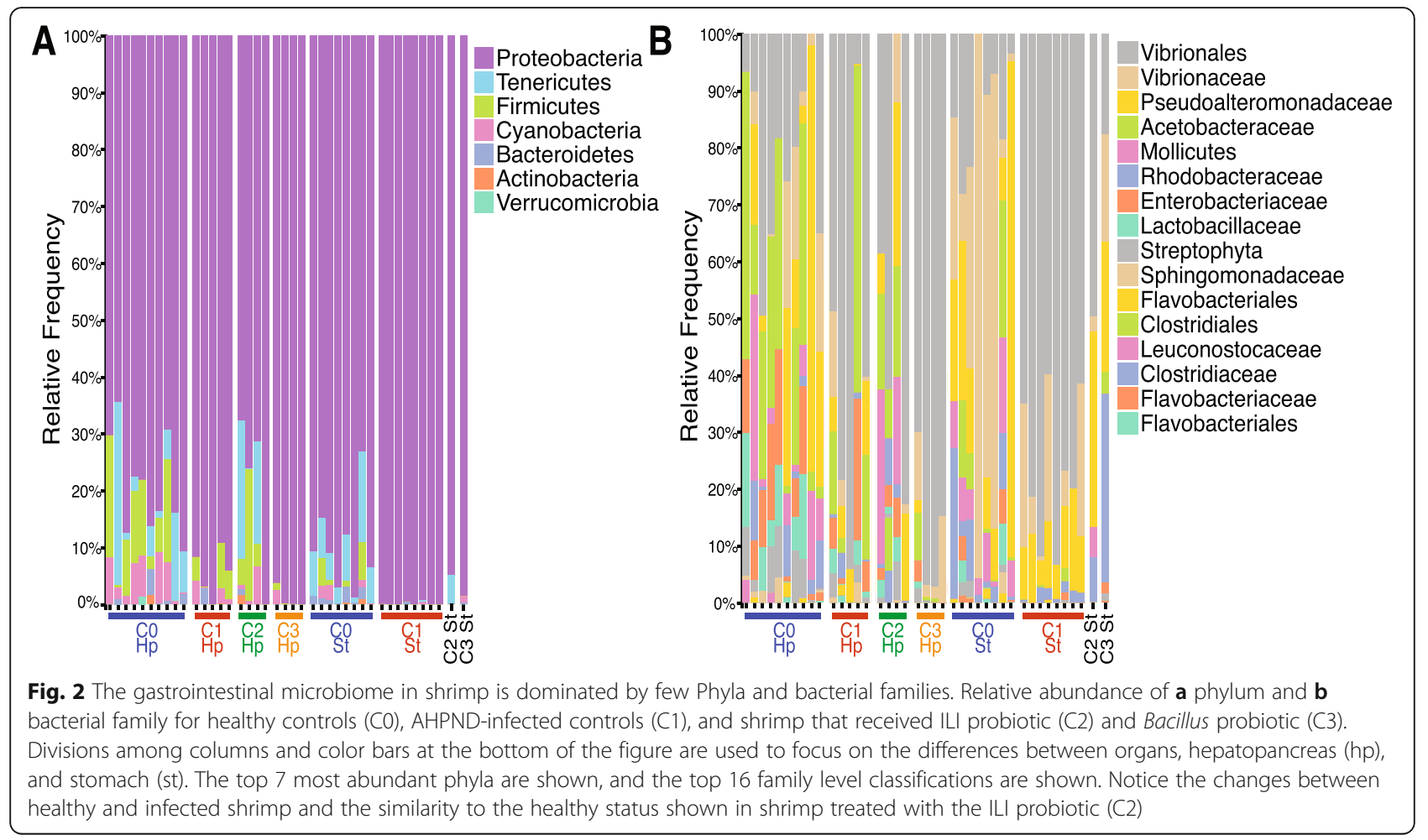

differentiate between known Vibrio clades. The three ASVs of Vibrionales were found within the cluster containing the species from the Orientalis, Coralliilyticus, and Harveyi clades (Fig. S3). A SNP in position 119 of the alignment showed differences between species of the three clades. Species that shared this SNP with the ASV for the Orientalis clade were Vibrio sinaloensis, Vibrio hepatarius, $V$. orientalis, and Vibrio brasiliensis; for Coralliilyticus clade, Vibrio neptunius; and finally, for the Harveyi clade, V. parahaemolyticus and V. campbellii.

Finally, we compared the nineteen ASVs that were differentially abundant in hepatopancreas and stomach samples of infected and healthy shrimp, as potential biomarkers in each of the microbiomes from the probiotic treatments. The most abundant ASVs characterized in hepatopancreas of AHPND-infected shrimp (Vibrionales 1 and Vibrionales 2) were found in different proportions among hepatopancreas microbiomes (Fig. S4A). The Rhodobacteraceae (ASV 3), Bacteriovorax sp. (ASV 4), and Ruegeria sp. (ASV 7) ASVs were found on AHPND-infected shrimp only, while Vibrionales 3 (ASV 5) was found both in AHPND-infected shrimp and shrimp treated with the Bacillus probiotic. When comparing ASVs as markers in stomach samples, we found that the four ASVs whose proportions were over-represented in infected organisms (Vibrionales 1, 2, 8, and 9) were stable across probiotic-treated animals, present at a comparable abundance as that in healthy animals. We observed ten ASVs in healthy shrimp: Nautella italica,

Table 2 Values (percent) of relative abundance of most abundant families for Healthy shrimp, AHPND-infected shrimp, and shrimp treated with the ILI and Bacillus probiotic

\begin{tabular}{|c|c|c|c|c|c|c|c|c|}
\hline \multicolumn{9}{|l|}{ Family level } \\
\hline & \multicolumn{2}{|c|}{ Healthy } & \multicolumn{2}{|c|}{ AHPND-infected } & \multicolumn{2}{|c|}{ Treated with the ILI probiotic } & \multicolumn{2}{|c|}{ Treated with the Bacillus probiotic } \\
\hline & $\mathrm{Hp}$ & St & $\mathrm{Hp}$ & St & $\mathrm{Hp}$ & St & $\mathrm{Hp}$ & St \\
\hline Vibrionales (Order) & 18.06 & 14.95 & 55.33 & 75.70 & 42.15 & 48.64 & 87.26 & 16.65 \\
\hline Pseudoalteromonadaceae & 15.94 & 21.70 & 5.75 & 10.31 & 13.53 & 32.94 & 0.58 & 21.63 \\
\hline Acetobacteraceae & 24.69 & 5.37 & 17.38 & 0.36 & 11.72 & 0 & 2.44 & 3.59 \\
\hline Rhodobacteraceae & 3.13 & 8.62 & 1.77 & 1.73 & 2.51 & 8.07 & 0.06 & 35.81 \\
\hline Enterobacteriaceae & 9.38 & 1.29 & 7.26 & 0.16 & 3.13 & 0 & 0.89 & 1.83 \\
\hline Mollicutes (Class) & 6.89 & 6.65 & 0 & 0 & 10.39 & 4.92 & 0 & 0 \\
\hline
\end{tabular}




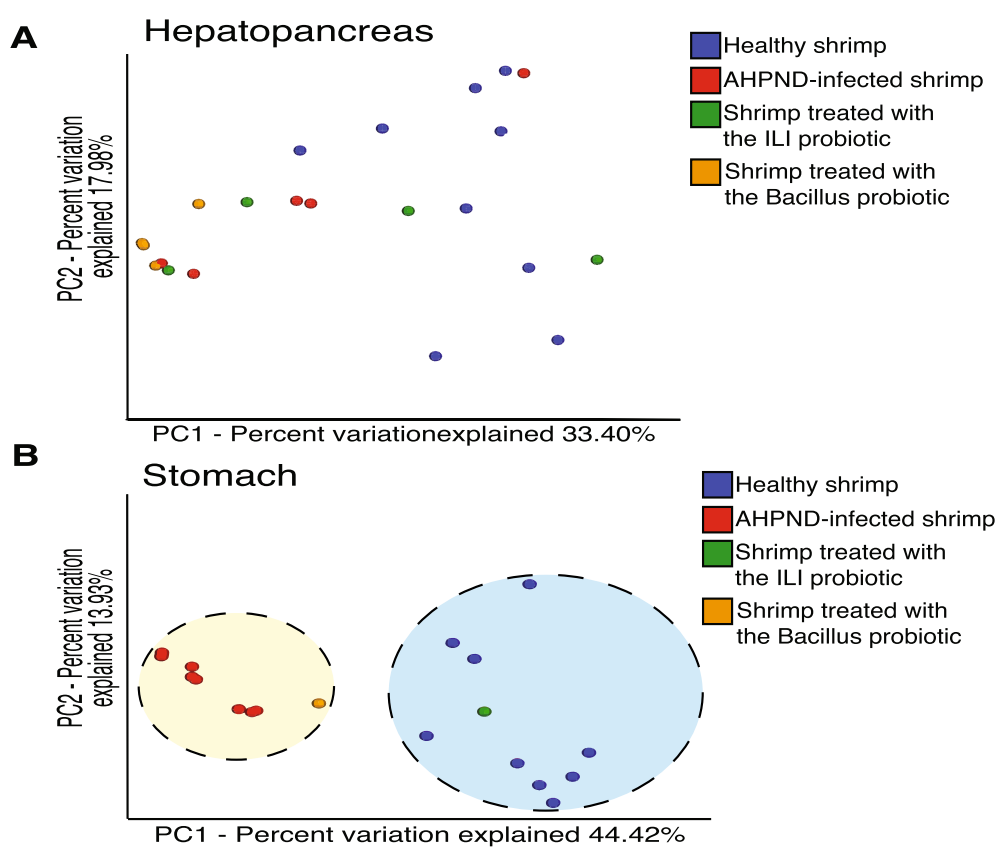

Fig. 3 Beta diversity analysis of microbiota from hepatopancreas and stomach samples. a A PCoA graphical representation based on Bray-Curtis similarity does not show a clear grouping pattern between different treatments for hepatopancreas samples in the two main axes explaining over $50 \%$ of the variation. b PCoA plot showing a clear grouping pattern that separates stomachs of infected from healthy samples. Variations between groups highlight the clustering of the ILI probiotic with the healthy shrimp, while the Bacillus probiotic seems closer to the infected individuals. Notice that all samples were ran in the same PCoA analysis but are separated by organ for visualization purposes. Each colored circle corresponds to a type of sample; healthy individuals (blue), infected individuals (red), with ILI probiotic (green) and Bacillus probiotic (orange). Probiotic treatments in panel B represent a pool of 5 stomachs processed together

Ruegeria, and Mollicutes 1 (ASVs from 10 to 12) were found in a higher proportion in healthy animals, while the Mollicutes 2, 3, and 4, Acetobacter and Photobacterium damselae (ASVs from 14 to 18) showed a significant increase in the ILI treatment as compared to healthy animals (Fig. S4B). The role of Photobacterium is unclear; it is known to inhabit many ecological niches and can perform a variety of functions related to host health [41], but it has also been associated with different diseases during shrimp farming [42]. An interesting idea may be to evaluate the role that this bacterium plays during AHPND infection. The finding of ten ASVs observed in healthy shrimp could be associated to the higher survival rate observed in shrimp treated with ILI. Tamlana crocina (ASV13) was found in higher proportions in probiotic treatments compared to controls; thus, it may be playing a role in shrimp's recovery.

\section{Bacterial bioassay shows that ILI probiotic strains do not cause AHPND in Penaeus vannamei shrimp}

Given the positive protective results obtained with the ILI probiotic strain and its close phylogenetic relationship with pathogenic strains, we decided to perform further genomic and experimental analyses on this strain. The $V$. alginolyticus ILI probiotic strain is a close relative of the pathogenic $V$. parahaemolyticus but does not contain the plasmid pV-AHPND with the Pir ${ }^{V P}$ genes coding for the AHPND-causing toxins PirA and PirB. We performed conjugation assays to evaluate the capacity of the ILI strain (recipient) to acquire the pV-AHPND plasmid from $V$. parahaemolyticus BA94C2 strain (donor). We found a high conjugation frequency of colonies harboring the plasmid carrying the Pir $^{V P}$ genes, with $70 \%$ of the evaluated ILI strain colonies containing the plasmid (Fig. 5a).

Transconjugant ILI colonies were isolated and used as the infecting agent for a challenge test with healthy $P$. vannamei shrimp, in order to evaluate their ability to cause AHPND in shrimp. Low mortality was observed for the transconjugant ILI treatment as well as negative controls (Fig. 5b). Shrimp mortality in the positive control (challenged with the $V$. parahaemolyticus BA94C2 strain) started after $6 \mathrm{~h}$ post-infection, reaching $96.2 \% \pm$ $0.5 \%$ at $50 \mathrm{~h}$ post-infection, resulting in a significantly different $(P=0.005$, Kruskal-Wallis test $)$ mortality compared to the transconjugated ILI strain treatment (Fig. 5b). Shrimp challenged with the transconjugant ILI strain were used for isolating predominant Vibrio strains. The recovered ILI strains were still positive for both PirA and PirB toxin genes. All Vibrio strains recovered from the macerates of the negative controls remained negative for the toxins (Fig. 5c). 


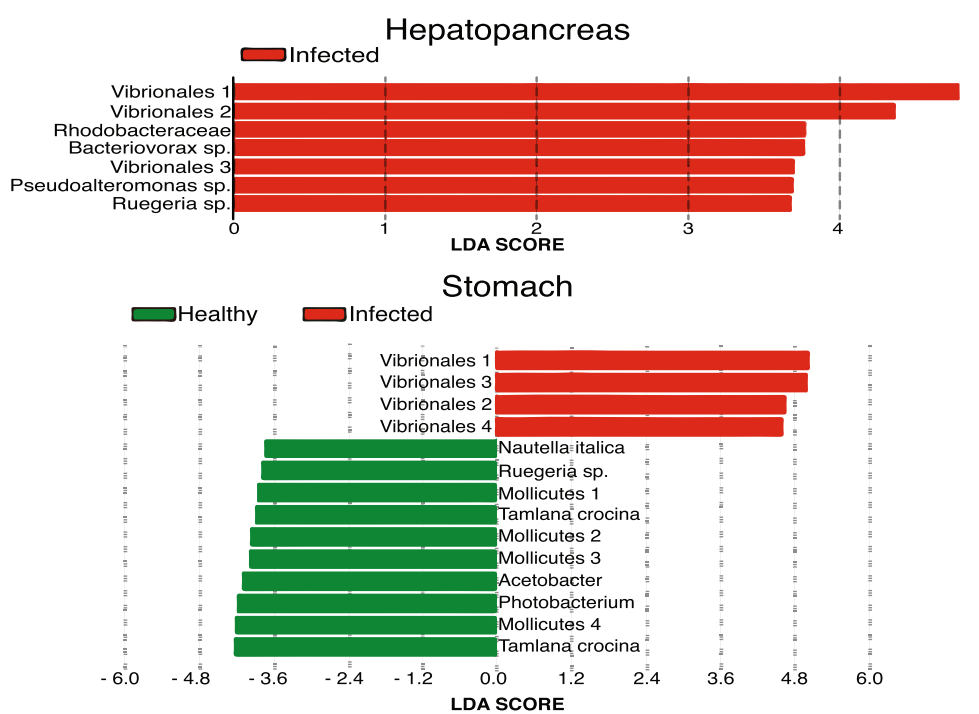

Fig. 4 LEfSe results of enriched ASVs of healthy and infected organisms. Enrichment was tested separately for ASVs in hepatopancreas (a) and stomachs (b). For the hepatopancreas, seven ASVs were the main contributors for the diversity differences observed and were only found enriched on infected individuals. For stomachs, fourteen ASVs showed differential abundance in AHPND-infected compared with healthy shrimps. The graph shows the log10 LDA score for each classification

\section{Genomic features of ILI probiotic strain and phylogenetic re-classification as Vibrio diabolicus}

Given its proven non-pathogenic capacity of the ILI probiotic strain in our essays, even after acquiring the $\mathrm{pV}$-AHPND, we aimed to understand the presence or lack of other potential virulence factors that could partly explain our findings. The genome of the ILI strain was sequenced using long reads (PacBio RS) and assembled into three scaffolds corresponding to chromosomes I (3.26 Mbp) and II (1.83 Mbp), and one large plasmid (340.52 Kbp, G+C content of $40.27 \%$ ), using a total of 82,331 PacBio reads. The average coverage achieved was $46.3 \mathrm{X}$, with $\mathrm{G}+\mathrm{C}$ content of $44.6 \%$. We predicted 4,956 coding genes (Table S3) within a total genome size of 5.4 Mbp (Table 4). Remarkably, the large plasmid not only had an unusually low $\mathrm{G}+\mathrm{C}$ content, but it did not show any significant similarity with plasmids previously described from Vibrio genomes available to date in public databases. The most closely related plasmid identified was described in $V$. campbellii strain 20130629003501 with $34 \%$ similarity, a size of $204,531 \mathrm{bp}$ and non-annotated function. Among the 496 plasmid proteins predicted by HMMER [43], only 96 (19\%) had significant matches in the NCBI non-redundant database (Table S2), leaving a total of $81 \%$ of proteins with unknown function.

The ILI probiotic strain was originally classified as $V$. alginolyticus [10] based on MLST analysis. We used the full genomic sequence and through comparisons against publicly available genomes (Table S4) showed that the closest significant matches are with Vibrio antiquarius EX25 (98\% identity) contrasting with a 92\% average identity against $V$. alginolyticus. To clarify the exact position of the ILI strain within the Harveyi clade, we used all available genomes of $V$. alginolyticus (31 genomes), Vibrio diabolicus (ten genomes) and $V$. antiquarius (two genomes) species, and two additional genomes of $V$. parahaemolyticus as an outgroup (Table S5). A concatenated alignment of seven genes ( $\mathrm{fts} Z$, gapA, mreB, recA, rpoB, topA, and $16 S$ rRNA) commonly used in MLST analysis was used to construct a maximum likelihood phylogenetic tree clearly separating $V$. alginolyticus from the $V$. diabolicus and $V$. antiquarius species in the Harveyi clade (Fig. 6). The phylogenetic tree revealed a high similarity level of the ILI strain with $V$. antiquarius and $V$. diabolicus, and four strains (E0666, FF273, TS13, and V2) previously identified as $V$. alginolyticus but recently reclassified as $V$. diabolicus by Turner et al. [44]. In that same study, it was suggested that $V$. diabolicus and $V$. antiquarius were the same species, and $V$. diabolicus should be used as it was described before $V$. antiquarius. Thus, our results clearly separate the ILI strain from the $V$. alginolyticus subclade and cluster this strain with others identified as the species $V$. diabolicus.

To further confirm this new classification, we took advantage of the availability of full genome sequences to perform genome-wide comparison against a selected set of representative isolates (Table S6), including AHPNDcausing and non-causing strains. The in silico-DDH (DNA-DNA hybridization) analysis showed that the ILI strain displayed a value higher than the traditional species delimiting cutoff (70\%) with $V$. antiquarius EX25 


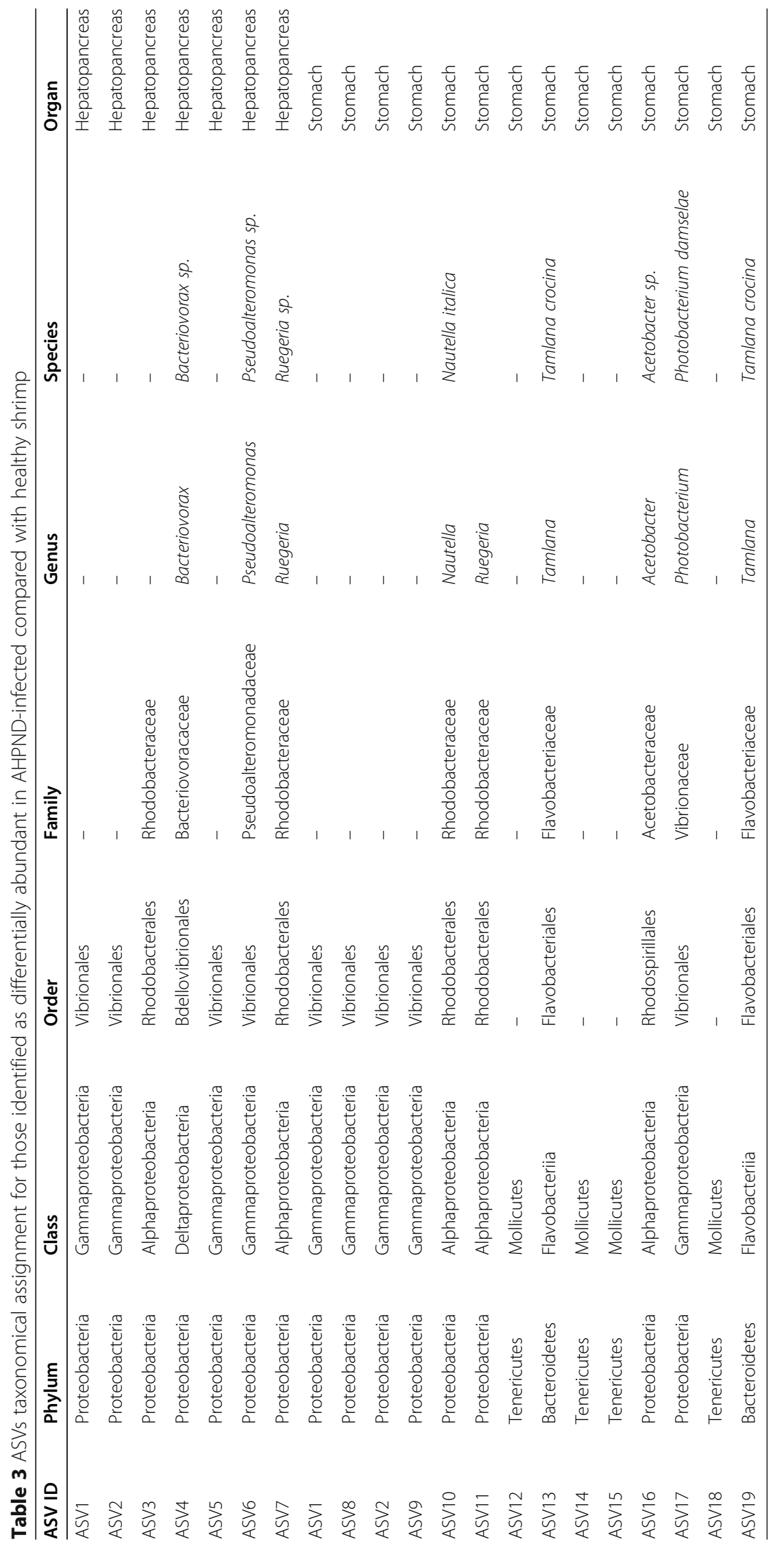




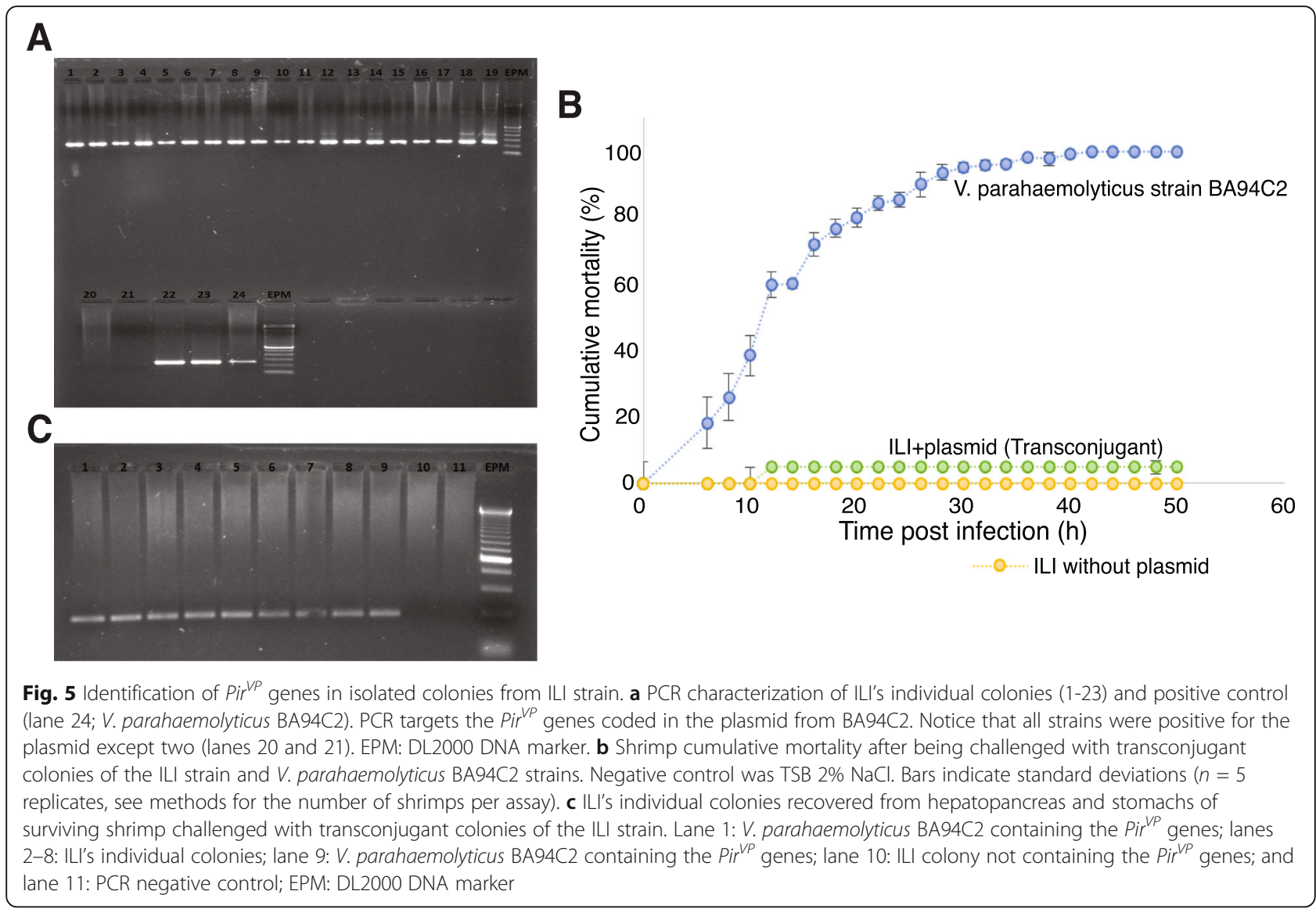

(83.80\%), and below the threshold with $V$. alginolyticus species $(\leq 46.30 \%$ Table S6). In addition, the percentage of differences of the $\mathrm{G}+\mathrm{C}$ content between ILI strain and $V$. antiquarius was $<1 \%$, corresponding to values obtained traditionally among members of the same species [45]. Phylogenomic metrics (ANI and Tetra) [43] were performed and the values between ILI and EX25 strain (reclassified as $V$. diabolicus, but still found in the nucleotide databases as $V$. antiquarius) was $98.03 \%$ for ANIb (Fig. 7a and Table S5). Accordingly, Tetra values indicated that the ILI strain is more related to EX25 strain based on the correlation coefficient of $99.8 \%$ (Fig. 7b). Based on the aforementioned results and the traditional thresholds of $95 \%$ and 0.997 for ANI and Tetra

Table 4 Genomic characteristics of the genome strain ILI

\begin{tabular}{ll}
\hline Attributes & Values \\
\hline Assembly size (bp) & $5,436,990$ \\
Total number of contigs & 3 \\
GC content \% & 44.6 \\
Number of CDS & 4,956 \\
Number of rRNAs & 18 \\
Number of tRNAs & 58 \\
\hline
\end{tabular}

to delimit the same species, here we reclassify the ILI strain as Vibrio diabolicus.

\section{Identification of specific virulence features of the $V$. diabolicus ILI strain genome}

The ILI genome contained 104 homologs of virulence genes found in other Vibrio species. Most of the homologs were classified in the subsystems of resistance to antibiotics and toxic compounds [46], which is interesting given the lack of pathogenicity shown in our experimental setups (Table S7). Furthermore, ILI's genome encodes a number of genes that include cytochrome peroxidase and cytochromes for reduction of oxygen to hydrogen peroxide and superoxide for tolerating high oxygen concentrations.

The presence of known secretion systems has been used to distinguish among $V$. parahaemolyticus pathogenic and non-pathogenic strains. In particular, $V$. parahaemolyticus contains two copies of type III secretion systems (T3SS1 and T3SS2), and two copies of type VI secretion systems (T6SS1 and T6SS2), where the numbering also signals their locations in chromosomes I and II, respectively. T3SS1 on ILI is also present in chromosome I and contains 43/49 genes (Table S7). In general, most of the genes show $98 \%$ average percent identity with the 


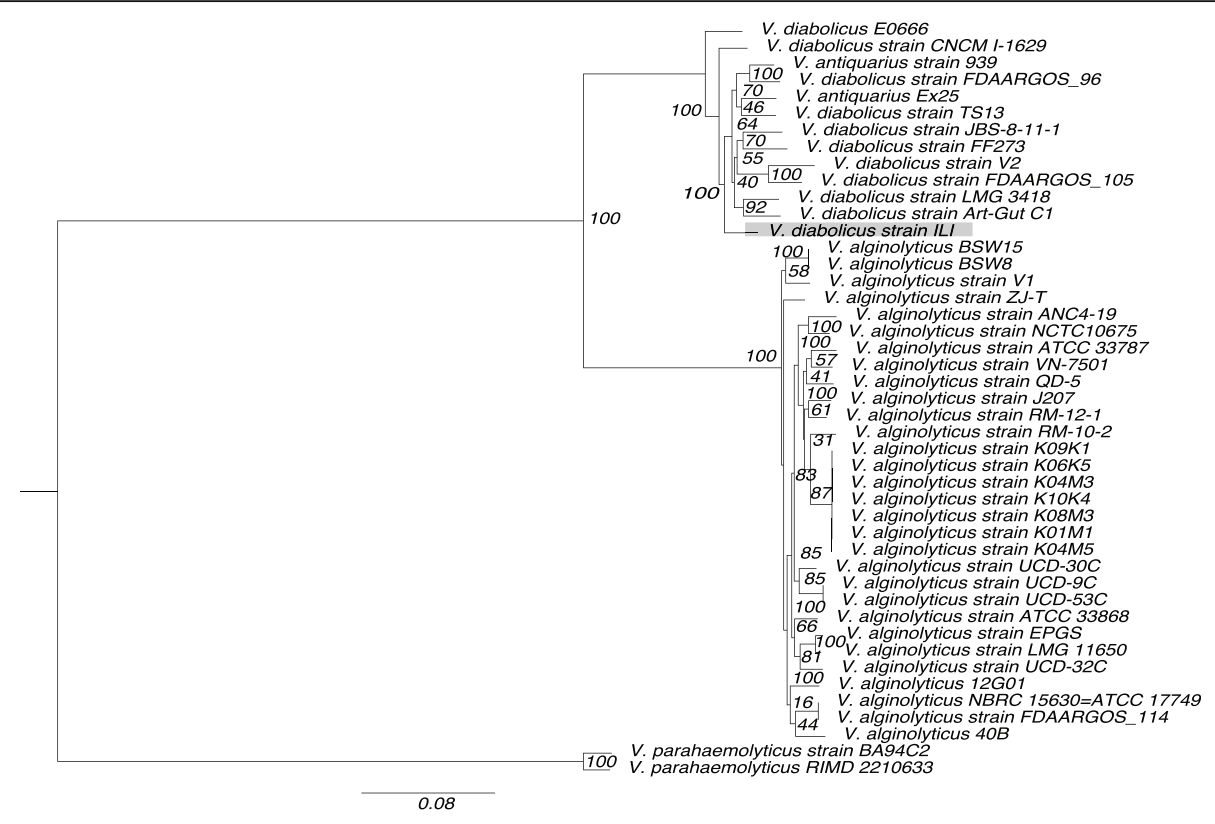

Fig. $6 \mathrm{ILI}$ strain reclassification as V. diabolicus. Phylogenetic analysis of the concatenated alignment based on ftsZ, gapA, mreB, recA, rpoB, topA, and 165 rRNA genes. A maximum likelihood phylogeny on a concatenated multiple sequence alignment using the TIM+G evolutionary model is shown. Numbers above the branches represent the posterior probabilities and percent bootstrap values (one thousand bootstrap replicates). The tree includes 10 Vibrio diabolicus strains, 20 Vibrio alginolyticus strains, 2 Vibrio antiquarius strains, and 2 Vibrio parahaemolyticus strains as the outgroup. Scale bar represents number of substitutions per site. Highlighted in grey is the position of the ILI strain

orthologs in $V$. alginolyticus and $V$. diabolicus, while only $84 \%$ with $V$. parahaemolyticus (Figure S4). In comparison, when evaluating 8 genes upstream and 9 genes downstream from T3SS1 in $V$. parahaemolyticus RIMD 2210633, a total of 15 out of 17 genes were present in ILI, with an average percent identity of $91.71 \%$.

T3SS2 is known to be present in clinical isolates but absent from environmental non-AHPND and toxic AHPND isolates of $V$. parahaemolyticus. It is composed by 12 genes and is located in chromosome II. Our results show that the ILI strain does not contain genes with an identity percentage greater than 50\%. An additional analysis of genes in the vicinity of T3SS2 resulted in the identification of only two downstream genes as VdILI_04698 and VdILI_04699, which are also located on chromosome II. Furthermore, is important to mention that none of the strains analyzed encode TDH/ TRH toxin on T3SS2, except for the clinical isolate RIMD2210633 (Table S7).

Regarding T6SS1 (42 genes), our analysis revealed that the non-AHPND isolates (30/42 genes for $V$. parahaemolyticus NCKU TN S02), a toxic AHPND isolate (34/42 genes for $V$. parahaemolyticus BA94C2), and the ILI strain contained at least partially the T6SS1 (34/42 genes), but in ILI is located on chromosome II, which is why we named it T6SS2B (Fig. 8; Table S7). This region was also found on chromosome II of the $V$. diabolicus strain FDA ARGOS 105. Salomon et al. [47] characterized the T6SS1 antibacterial activity of the RIMD2210633 strain and showed that it is mediated by at least three effectors. We further searched for the three Vibrio parahaemolyticus (VP) effectors of T6SS1 and were able to identify them in ILI's genome (Fig. 8): $V$. parahaemolyticus RIMD2210633_01364 (VdILI_ 03751, Identity $99.21 \%$, Coverage $100 \%$ ) and $V$. parahaemolyticus RIMD2210633_01391 (VdILI_03724, Identity 91.98\%, Coverage 19\%) within the T6SS1 and effector $V$. parahaemolyticus RIMD2210633_01397 (VdILI_03720, Identity $96.43 \%$, Coverage 91\%) was present in chromosome I.

The T6SS2 (containing 22 genes, $V$. parahaemolyticus RIMD2210633_040- V. parahaemolyticus RIMD2210633_ 04059) is also highly conserved in $V$. parahaemolyticus; it is located on chromosome II and found in pathogenic and non-pathogenic isolates of $V$. parahaemolyticus (Table S7). An equivalent cluster with a high number of genes (20/22) was identified on $V$. diabolicus ILI strain on chromosome II (named T6SS2A); the genes were present in ILI with an average percent identity of $73.29 \%$. In comparison, when evaluating 10 genes upstream and downstream from T6SS2A in $V$. parahaemolyticus RIMD 2210633, a total of 14/20 genes were present in ILI with an average percent identity of $86.69 \%$. 


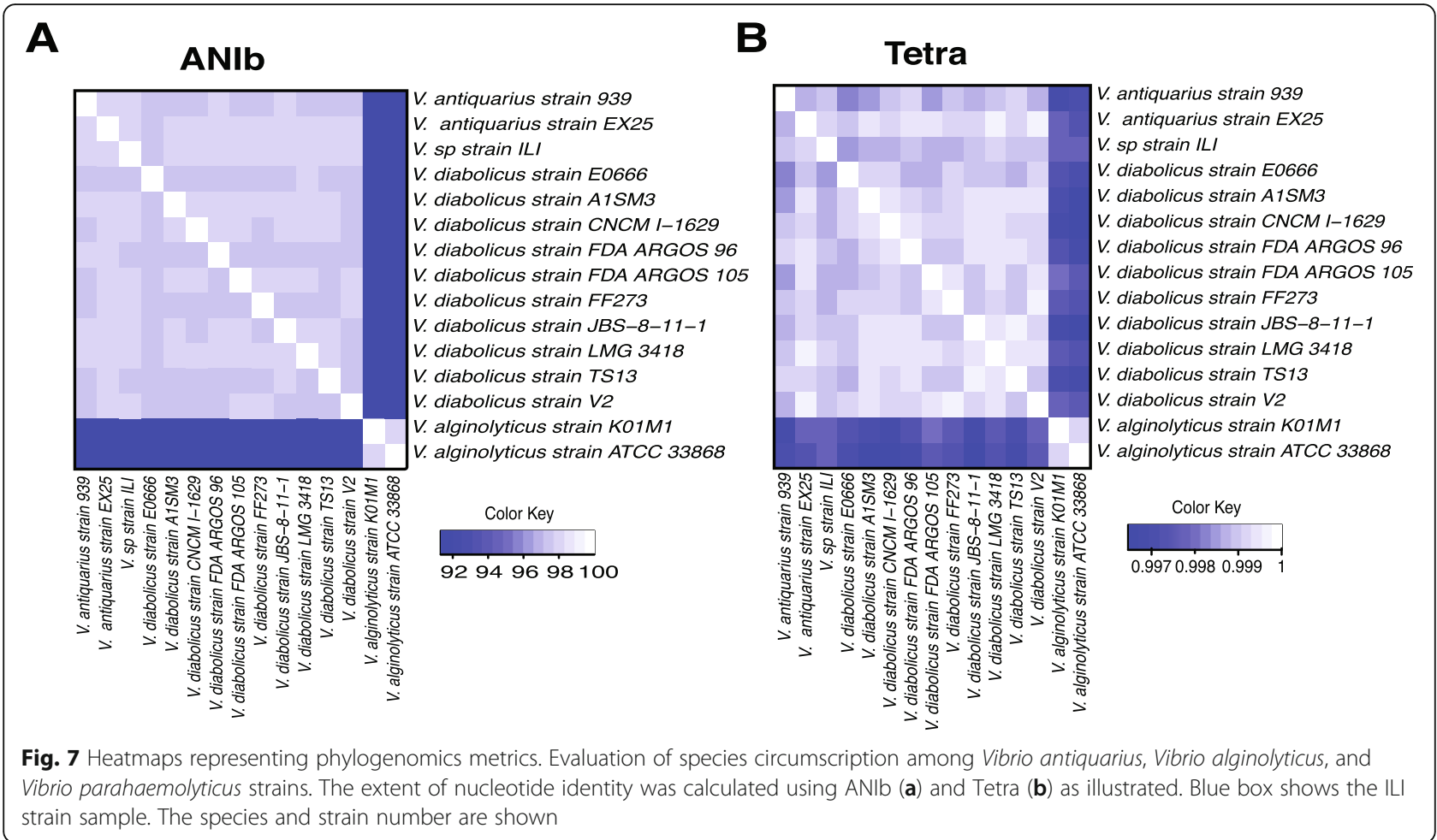

\section{Discussion}

Shrimp is one of the most important products for aquaculture worldwide. However, due to the massive scale in which shrimp farming is performed, it becomes difficult to control for environmental variables, in particular the presence of pathogens. Currently, AHPND is one of the major risk factors in shrimp farming [2]. Methods such as antibiotics prove to be expensive and unspecific targeting beneficial bacteria as well; thus, probiotics are an important alternative to combat infectious diseases. To date there is very limited information of the effect of AHPND in the shrimp microbiome [48], and existing studies have focused on intestinal microbiota. Even though the contribution of intestinal microbiota on the impact of AHPND disease is not clear, the authors highlight the fact that the diversity on the intestine is higher than on the hepatopancreas, but similar to the one found in sediment. It is known that the immune related organs such as hemocytes, hepatopancreas and gills, are important in the response to AHPND disease [49]. Also, the colonization of the stomach appeared to be the initial step of the infection for Vibrio causing AHPND [49]. Additionally, AHPND mainly affects the hepatopancreas and stomachs of infected shrimp; nevertheless, characterizing the microbiota from the shrimp's stomach has not been a main focus to understand the changes in microbiota responses. Therefore, we examined microbiome alterations in response to pathogenic bacteria producing AHPND, in stomach and hepatopancreas of shrimp.
We identified a significant loss of diversity in the microbiota of hepatopancreas and stomach associated with AHPND $(P<0.001$, Kruskal-Wallis test). Our results contrast with some minimal effects observed in the intestinal microbiota of shrimp after WSSV infection [50] and AHPND [48]. Thus, we consider that the abundance and composition of the microbiota are influenced as a function of the organ in which it is located. When looking at the effect of the probiotics in the community structure, we observed that the microbiota was closer to the healthy state than the infected configuration of the microbiome, with ILI being the most similar to the microbiota of healthy animals, maintaining a phylumlevel diversity including Proteobacteria, Firmicutes, and Tenericutes, which are also the main components of intestinal microbiome in other species, such as crab and black tiger shrimp [50,51]. In this study, we show that in AHPND infected shrimp, Proteobacteria (primarily order Gammaproteobacteria) was the most dominant phylum and increased in abundance when compared to healthy shrimp and the ILI probiotic treatment.

Interestingly, we identified members from the Tenericutes phylum, in particular those assigned to the genus Mycoplasmatales, as members of the healthy microbiome. Four ASVs from Mycoplasmatales belonged to the genus Mycoplasma, being the most abundant taxonomic group of the healthy stomach's microbiome. This taxon is a common microbiome member in corals like scleractinians and gorgonians [52, 53]. Studies on the 


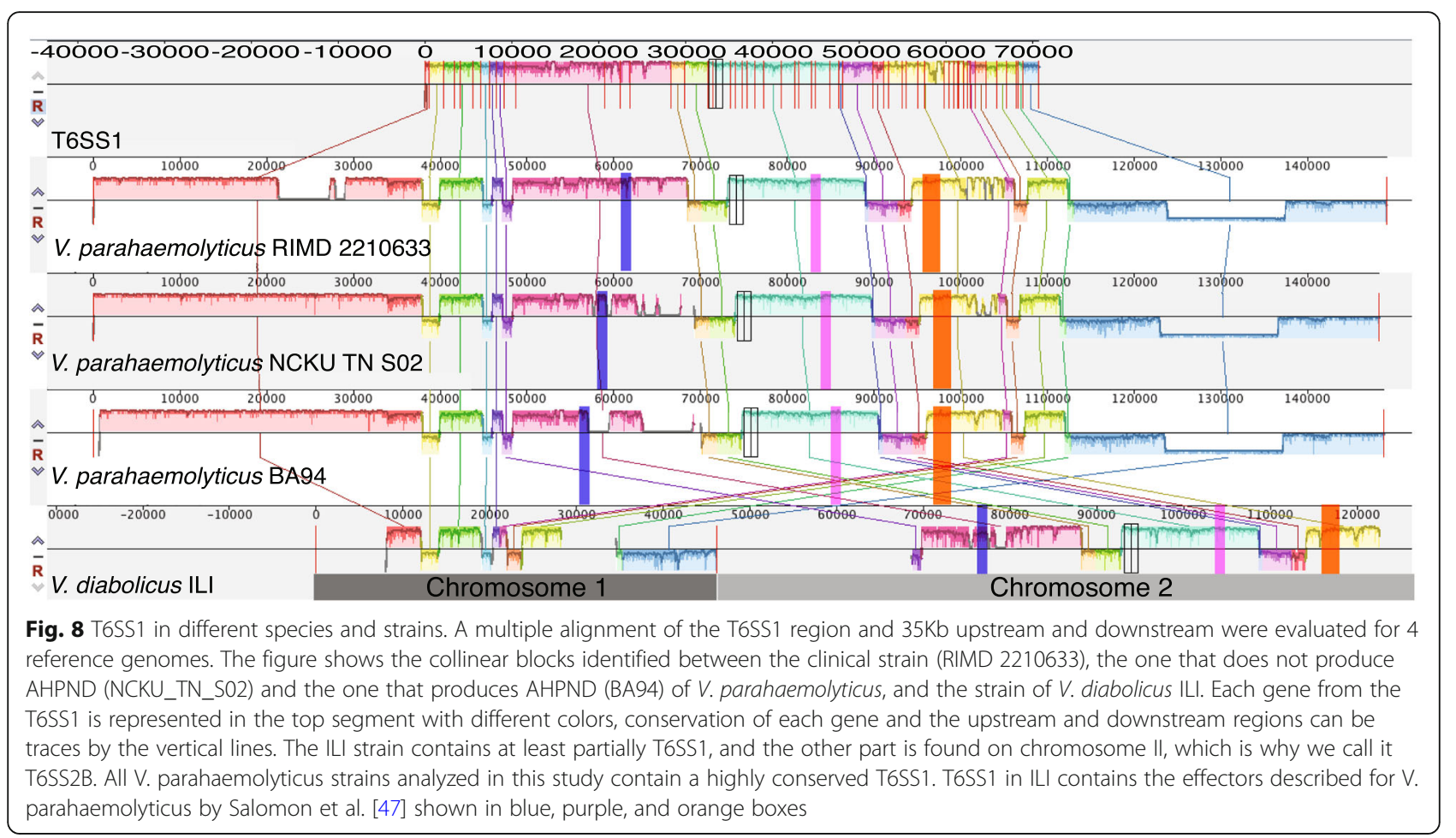

role of Mycoplasma spp. in corals and in some cnidarians $[52,54]$ have suggested that it would be harmless commensals or endosymbionts with the host; however, its specific role within the shrimp microbiome is unknown. Specific strains of Mycoplasma have been associated with healthy shrimp $[55,56]$, potentially suggesting that it has a function in host health and recovery from disease states.

We observed an increase in Proteobacteria in infected animals, in particular, most ASVs associated with disease were associated with the Vibrionales order, mainly the Vibrionaceae family. Given that $16 S$ rRNA gene sequences have a high degree of similarity within the Vibrionaceae, it is impossible to discriminate with precision which ASVs are associated with the pathogen ( $V$. parahaemolyticus) or with the probiotic (ILI strain) or any other Vibrio present in the microbiome. However, the presence of multiple ASVs associated to this family suggest that not only the pathogenic strain responsible of the disease is increasing in abundance, but potentially other taxonomically related Vibrios are increasing as well in consequence of the niche made available by the pathogenic strain. The alignments of ASVs suggested that Vibrionales 1 and 2 can be used as the major biomarkers in the microbiomes of the AHPND-infected shrimp in hepatopancreas, due to the close relationship with species that have been characterized as pathogenic, specifically in the Harveyi clade; while Mollicutes species could be used as a potential indicator of health or recovery in microbiomes isolated from individual shrimp stomachs.

Surprisingly, some members of the order Vibrionales and Rhodospirillales appeared as associated to healthy individuals. Some of these bacteria have shown antagonistic effects against different bacterial pathogens including known coral pathogens as Vibrio sp. [52]. Rhodospirillales species have been reported as probiotics before, but this could demonstrate that these ASVs could be protective against pathogenic Vibrio and that it could improve the survival and immunity of shrimp. Furthermore, we were able to observe that the use of bacterial probiotics, such as ILI, can modulate the host microbiota and could contribute as a stimulant for several species that have probiotic potential by inhibiting gastrointestinal colonization of pathogens, as found in this study.

The significant protective effect that the ILI strain showed, and its similarity, at least by $16 S$ rRNA gene sequence, to potential pathogens, led us to further study the genomic composition and phylogenetic relatedness of this strain. The comparative genomics approach used confirmed inconsistencies in the species classification, where not only the ILI strain was mistakenly classified but other strains had similar errors, likely due to the lack of complete genomic information at the time when the species were described. Current genomic tools and available genomes allowed us to classify them as $V$. diabolicus. 
The genomic repertoire of ILI's genome includes cytochrome peroxidase and other cytochromes for reduction of oxygen to hydrogen peroxide, and superoxide for tolerating high oxygen concentrations, and multiple catalase genes. For example, the Bcp-type encodes a functional thiol peroxidase and plays a role in the precise control of $\mathrm{H}_{2} \mathrm{O}_{2}$ levels. The $\mathrm{H}_{2} \mathrm{O}_{2}$ serves as an intracellular messenger at low concentrations, and it induces cell death at higher concentrations. Besides, the host's immune system will use reactive oxygen species against pathogenic bacteria, which shows the bacteria's need for an adequate detoxifying system [57]. These results are compatible with the ones reported by Hasan et al. [58], where the authors suggested that the ability to scavenge endogenous hydrogen peroxide was absent in the other Vibrio genomes but was present in the genome of $V$. antiquarius EX25. Furthermore, Yévenes et al. [59] reported that Vibrio sp. ArtGut-C1 strain has the ability to accumulate polyhydroxybutyrate (PHB), suggesting that $V$. diabolicus, $V$. antiquarius, and $V$. alginolyticus could potentially produce the polymer as they also have PHB genes. It is an interesting finding given that this biodegradable polymer could have anti-pathogenic activity, particularly in the marine larviculture phase. Sequencing of the genome identified genes that confer resistance to antibiotics, in addition to genes that improve the fitness of the organism.

The fact that ILI can acquire the causative plasmid for AHPND shows the high capacity of Vibrio species for HGT, which may be used to occupy different niches. The acquisition may vary in the aquatic environment and it may be influenced by the genetic background. ILI strain harbors hemolysins, T3SS, and T6SS that are considered factors associated with virulence in pathogenic Vibrio species. We found a certain level of presence and conservation of such secretion systems in ILI; however, the variation compared to the pathogenic strains prevents us from concluding regarding the functionality of such systems. The maintenance of these features in nonpathogenic bacteria could be evidence that secreted toxins are evolutionarily ancient features that may play a larger role in environmental fitness [58].

Pathogens use a specialized set of T3SS translocator proteins to establish virulence in the host cell since it directly delivers bacterial virulence factors, called effectors, into the cytoplasm of host cells in order to exert various functions [60]. Wang et al. [61] determined that four effectors (Vop Q, Vop S, VPA0450 and Vop R (VP1638)) located on T3SS1 of $V$. parahaemolyticus are involved in the cytotoxic process, causing autophagy, cell rounding, and finally death. Even though the main cluster of T3SS1 in the ILI strain is structurally similar to the one previously characterized in $V$. parahaemolyticus, it does not possess the four effectors described before (Table S7).
Similarly, a homology analysis indicated that the T6SS1 effectors (VdILI_03751, VdILI_03724 and VdILI_ 03720) are present in different Vibrio species, including $V$. alginolyticus, $V$. campbellii, and $V$. cholerae [62]. These effectors were described as necessary for the adhesion of $V$. parahaemolyticus to cells and are involved in intracellular trafficking and vesicular transport. The three effectors contain the conserved MIX motif that is found in proteins with predicted cytotoxic domains, including VgrG and PAAR-repeat-containing protein [63]. $\mathrm{Li}$ et al. [18] evaluated whether T6SS2 (equivalent toT6SS2A in ILI strain) activates under similar conditions in all types of bacterial strains tested and found that various strains tested under the same conditions did not show T6SS2 activity. They concluded that besides temperature, activation can be linked to other factors. Furthermore, both T6SSs have been defined as required for virulence or survival of a bacterium in a eukaryotic host. However, Weber et al. [64] reported that T6SS in $V$. anguillarum regulates stress response, suggesting that T6SS has an ecological function besides other potential pathological function, as can be the case of the ILI strain. Thus, altogether, our data indicates that virulence is multifactorial in the Harveyi clade and the sources of virulence could be associated to specific hosts since many of these genes can be involved on the survival of the bacteria in different environments.

The ILI plasmid (340 Kbp) has not been described in any previous report, but it could provide important functions in the probiotic capacities that this strain exhibits. It is intriguing that no other reported pathogenic Vibrio strain contains a plasmid with similar gene content. The majority of large plasmids that are associated with Vibrio species have been characterized to be highly virulent against different hosts. Interestingly, we did not find any potential virulence factors encoded in the ILI plasmid; thus, the role of the plasmid in the bacteria is not yet clear. Further studies on the role of the plasmid in the ILI strain could clarify its function and potentially shed light on whether it contributes to ILI's probiotic capabilities.

\section{Conclusions}

The use of probiotics in aquaculture is a practical alternative to promote animal health and prevent disease. We characterized the microbiome alterations generated by a successful probiotic that could control pathogenic populations in shrimp's gastrointestinal tract and stimulate survival in aquaculture. $V$. diabolicus ILI strain is a bacterial strain isolated from the environment in a larvae shrimp culture that has shown antimicrobial activity against many pathogenic strains of different Vibrio species [13]. Supplementation of ILI to P. vannamei not only decreased the abundance of Proteobacteria ASVs, 
but also maintained a healthy-like microbial community in the shrimp gastrointestinal tract after being challenged with AHPND causing bacteria. Our findings suggest that ILI strain can likely be used as a probiotic to reduce the population of pathogens for AHPND in the shrimp and to enhance survival and resistance against this emerging disease in shrimp aquaculture, without the risk of becoming a pathogenic organism.

\section{Supplementary Information}

The online version contains supplementary material available at https://doi. org/10.1186/s40168-021-01043-8.

Additional file 1: Figure S1. Shrimp cumulative mortalities after feeding the animals with the probiotics for a month's period and being challenged with Vibrio parahaemolyticus BA94C2 strain. Negative control was TSB 2\% NaCl. Mortalities were followed for up to 3 days. It is observed a delay on the onset of mortalities and a reduction in cumulative mortality as an effect of the probiotic treatment.

Additional file 2: Figure S2. Rarefaction curves for samples obtained from all treatments. Graphs represent the Observed OTU metric subsampled at different sequencing depths $(500-4000)$ with 10 different replicates. Saturation is shown at approximately 1500 sequences for all samples.

Additional file 3: Figure S3. Graphical visualization of the alignment of V4 region of the 165 rRNA gene from 38 related Vibrio species. Variations from the reference sequence are highlighted along the length of the alignment for species of the different known Vibrio species. Although taxonomical assignments would assign four ASVs to the order Vibrionales, it is possible to observe clade specific SNPs in particular location of the sequences. The set of ASV recovered from the microbiota experiment are highlighted in red.

Additional file 4: Figure S4. Evaluation of possible biomarkers. The abundances of ASVs in the stomach of infected, healthy, and probiotictreated shrimp were compared. (A) The ASVs identified as discriminatory for AHPND-infected shrimp in the stomach were Vibrionales 1, 3, 2 and 4. (B) The ASVs identified as discriminatory for healthy shrimp in the stomach were Mollicutes 2 and 3, Acetobacter and Photobacterium damselae. Interestingly, we found that these ASVs showed a significant increase in ILI treatment compared to healthy animals. Samples from C0 and C1 correspond to 5 samples, while for C2 and C3 is a single pooled sample.

Additional file 5: Table S1. Primers sequences used in the study. AP4 (1 and 2) are for the detection of Pir-VP genes. Vpir A is for the detection of Pir-A gene while Vpir B is for the amplification of Pir-B gene. Length represents the length of the amplicon generated by each pair of primers Table S2. ILI's genome protein annotation using Prokka against the NCBI's database. Table S3. ILI's large plasmid proteins predicted by Prokka against the NCBI's database with their corresponding functions and ID. The number of ASVs detected per sample is plotted on the axis of Observed OTUs. Function is assigned when hits with an e-value $<1$ 06 were obtained. Table S4. Genomes used to for genomic similarity analysis. The genomic identity between ILI and the selected genome is shown in the last column of the table. Table S5. GenBank accession numbers and genomic characteristics of genomes used for the phylogenetic reconstruction of the ILI strain. Table S6. DDH, ANIb and TETRA values. In silico DNA-DNA hybridization (DDH) was calculated only between the ILI strain and the other evaluated strains. ANIb and TETRA were calculated for all possible comparisons. A heatmap representation of the values can be observed on Figure 3. Table S7. Virulence factors identified in the secretion systems of the strain V. diabolicus ILI. They were compared with the genomes of the $V$. parahaemolyticus RIMD 2210633 strain, the $V$. parahaemolyticus NCKU TN S02 strain, the $V$. parahaemolyticus BA94C2 strain and the $V$. diabolicus FDA 105 strain. The table includes the respective name of the genes for each genome, the gene product, the percent identity, and the percent coverage. Comparisons based on percentage of identity and coverage included are shown in key color.

\section{Acknowledgements}

We thank Juan Carlos Muñoz for his technical assistance in this study. We would like to thank Luis Alberto Chica, David Ayala, and Laura Avellaneda for their valuable advice on data analysis and Dr. Stanislaus Sonnenholzner and Dr. Maryam Chaib for their kind reviewing of the manuscript and suggestions. Additionally, we acknowledge the High-Performance Computing Service at Universidad de los Andes in Bogotá, Colombia, (URL: http:// hpc.uniandes.edu.co) for providing HPC resources that have contributed to the research results reported in this work.

\section{Authors' contributions}

Conceptualization: $L R, J R, B B, A R$. Data curation and formal analysis: $L R, L B, I B$. Funding acquisition: $B B$. Investigation: $L R, C D B, L B$, IB. Project administration: BB. Resources: BB, AR. Supervision: BB, AR. Writing-original draft: LR. Writing — review and editing: all. The author(s) read and approved the final manuscript.

\section{Funding}

This work was funded by the Secretaria de Educación Superior, Ciencia, Tecnología e Innovación (SENESCYT) in the framework of the PIC-14-CENA IM-003 Project "Desarrollo e implementación de métodos de control y prevención de enfermedades en especies acuáticas de uso comercial y uso potencial en maricultura y repoblación."

\section{Availability of data and materials}

All relevant data are within the paper and its additional files. Raw sequence data from this experiment were uploaded to the Sequence Read Archive (SRA) under Bioproject accession number PRJNA580262.

\section{Declarations}

Ethics approval and consent to participate

This study does not involve the use of human subjects. The ethical statements regarding animal experimentation are included in the "Materials and methods" section.

\section{Consent for publication}

Not applicable.

\section{Competing interests}

The authors declare that they have no competing.

\section{Author details}

${ }^{1}$ Department of Biological Sciences, Universidad de los Andes, Bogotá, Colombia. ${ }^{2}$ Max Planck Tandem Group in Computational Biology, Universidad de los Andes, Bogotá, Colombia. ${ }^{3}$ Escuela Superior Politécnica del Litoral, ESPOL, Centro Nacional de Acuicultura e Investigaciones Marinas, CENAIM, Campus Gustavo Galindo Km 30.5 Vía Perimetral, P.O. Box 09-01-5863, Guayaquil, Ecuador. ${ }^{4}$ Escuela Superior Politécnica del Litoral, ESPOL, Facultad de Ciencias de la Vida, FCV, Campus Gustavo Galindo Km 30.5 Vía Perimetral, P.O. Box 09-01-5863, Guayaquil, Ecuador. ${ }^{5}$ Escuela Superior Politécnica del Litoral, ESPOL, Facultad de Ingeniería Marítima y Ciencias del Mar, FIMCM, Campus Gustavo Galindo Km 30.5 Vía Perimetral, P.O. Box 09-01-5863, Guayaquil, Ecuador. ${ }^{6}$ Center for Genome Sciences and Systems Biology, Department of Pathology and Immunology, Washington University in Saint Louis, Saint Louis, MO, USA.

Received: 7 December 2020 Accepted: 5 March 2021

Published online: 12 April 2021

\section{References}

1. Defoirdt T, Sorgeloos P, Bossier P. Alternatives to antibiotics for the control of bacterial disease in aquaculture. Curr Opin Microbiol. 2011;14(3):251-8. https://doi.org/10.1016/j.mib.2011.03.004.

2. Hong $X, L u ~ L, X u$ D. Progress in research on acute hepatopancreatic necrosis disease (AHPND). Aquac Int. 2016;24(2):577-93. https://doi.org/10.1 007/s10499-015-9948-X.

3. Devadas S, Banerjee S, Yusoff FM, Bhassu S, Shariff M. Experimental methodologies and diagnostic procedures for acute hepatopancreatic necrosis disease (AHPND). Aquaculture. 2019;499:389-400. https://doi.org/1 0.1016/j.aquaculture.2018.06.042. 
4. Lee CT, Chen IT, Yang YT, Ko TP, Huang YT, Huang JY, Huang MF, Lin SJ, Chen CY, Lin SS, Lightner DV, Wang HC, Wang AHJ, Wang HC, Hor LI, Lo CF. The opportunistic marine pathogen Vibrio parahaemolyticus becomes virulent by acquiring a plasmid that expresses a deadly toxin. PNAS. 2015; 112(34):10798-803. https://doi.org/10.1073/pnas.1503129112.

5. Tran L, Nunan L, Redman RM, Mohney LL, Pantoja CR, Fitzsimmons K Lightner DV. Determination of the infectious nature of the agent of acute hepatopancreatic necrosis syndrome affecting penaeid shrimp. Dis Aquat Org. 2013;105(1):45-55. https://doi.org/10.3354/dao02621.

6. Waterfield N, George Kamita S, Hammock BD, Ffrench-Constant R. The Photorhabdus Pir toxins are similar to a developmentally regulated insect protein but show no juvenile hormone esterase activity. FEMS Microbiol Lett. 2005;245(1):47-52. https://doi.org/10.1016/j.femsle.2005.02.018.

7. Cabello FC. Heavy use of prophylactic antibiotics in aquaculture: a growing problem for human and animal health and for the environment. Environ Microbiol. 2006;8(7):1137-44. https://doi.org/1 $0.1111 /$ j.1462-2920.2006.01054.x

8. Okocha RC, Olatoyel O, Adedeji OB. Food safety impacts of antimicrobial use and their residues in aquaculture. Public Health Rev. 2018;39(1):1-22

9. Suez J, Zmora N, Zilberman-Schapira G, Mor U, Dori-Bachash M, Bashiardes S, Zur M, Regev-Lehavi D, Ben-Zeev Brik R, Federici S, Horn M, Cohen Y, Moor AE, Zeevi D, Korem T, Kotler E, Harmelin A, Itzkovitz S, Maharshak N, Shibolet O, Pevsner-Fischer M, Shapiro H, Sharon I, Halpern Z, Segal E, Elinav E. Post-antibiotic gut mucosal microbiome reconstitution is impaired by probiotics and improved by autologous FMT. Cell. 2018;174(6):1406-23. https://doi.org/10.1016/j.cell.2018.08.047.

10. De Wolfe TJ, Eggers S, Barker AK, Kates AE, Dill-McFarland KA, Suen G, Safdar N. Oral probiotic combination of Lactobacillus and Bifidobacterium alters the gastrointestinal microbiota during antibiotic treatment for Clostridium difficile infection. PLoS One. 2018;13(9):e0204253. https://doi.org/10.1371/ journal.pone.0204253.

11. Pinoargote G, Flores G, Cooper K, Ravishankar S. Effects on survival and bacterial community composition of the aquaculture water and gastrointestinal tract of shrimp (Litopenaeus vannamei) exposed to probiotic treatments after an induced infection of acute hepatopancreatic necrosis disease. Aquac Res. 2018;49(10):3270-88. https://doi.org/10.1111/are.13791.

12. Rodríguez J, Espinosa Y, Echeverría F, Cárdenas G, Román R, Stern S. Exposure to probiotics and $\beta-1,3 / 1,6$-glucans in larviculture modifies the immune response of Penaeus vannamei juveniles and both the survival to White Spot Syndrome Virus challenge and pond culture. Aquaculture. 2007; 273(4):405-15. https://doi.org/10.1016/j.aquaculture.2007.10.042.

13. Vandenberghe J, Verdonck L, Robles-Arozarena R, Rivera G, Bolland A, Balladares M, Gomez-Gil B, Calderon J, Sorgeloos P, Swings J. Vibrios associated with Litopenaeus vannamei larvae, postlarvae, broodstock, and hatchery probionts. Appl Environ Microbiol. 1999;65(6):2592-7. https://doi. org/10.1128/AEM.65.6.2592-2597.1999.

14. Rodríguez J, Cedeño R, Bayot B, Echeverría F, da Silva JA, Valladares A, et al. Effects of the Vibrio alginolyticus probiotic, E-1, 3/1, 6-glucans and temperature on shrimp production. Dyn Biochem Process Biotechnol Mol Biol. 2011;5:49-55.

15. Gullian M, Thompson F, Rodriguez J. Selection of probiotic bacteria and study of their immunostimulatory effect in Penaeus vannamei. Aquaculture. 2004;233(1-4):1-14. https://doi.org/10.1016/j.aquaculture.2003.09.013.

16. Sotomayor MA, Reyes JK, Restrepo L, Domínguez-Borbor C, Maldonado M, Bayot B. Efficacy assessment of commercially available natural products and antibiotics, commonly used for mitigation of pathogenic Vibrio outbreaks in Ecuadorian Penaeus (Litopenaeus) vannamei hatcheries. PLoS One. 2019; 14(1):e0210478. https://doi.org/10.1371/journal.pone.0210478.

17. Restrepo L, Bayot B, Arciniegas S, Bajaña L, Betancourt I, Panchana F, Muñoz AR. Pir ${ }^{V P}$ genes causing AHPND identified in a new Vibrio species (Vibrio punensis) within the commensal Orientalis clade. Sci Rep. 2018:8(1):13080. https://doi.org/10.1038/s41598-018-30903-x.

18. Li P, Kinch LN, Ray A, Dalia AB, Cong Q, Nunan LM, et al. Acute hepatopancreatic necrosis disease-causing Vibrio parahaemolyticus strains maintain an antibacterial type VI secretion system with versatile effector repertoires. Appl Environ Microbiol. 2017;83(13):e00737-17.

19. Raghunath $P$. Roles of thermostable direct hemolysin (TDH) and TDH-related hemolysin (TRH) in Vibrio parahaemolyticus. Front Microbiol. 2015;5:805.

20. Llosa M, Bolland S, de la Cruz F. Structural and functional analysis of the origin of conjugal transfer of the broad-host-range IneW plasmid R388 and comparison with the related IncN plasmid R46. MGG. 1991;226(3):473-83. https://doi.org/10.1007/BF00260661.

21. Wang YI, Naumann U, Wright ST, Warton DI. mvabund-an R package for model-based analysis of multivariate abundance data. Methods Ecol Evol. 2012;3(3):471-4. https://doi.org/10.1111/j.2041-210X.2012.00190.x.

22. Caporaso JG, Lauber CL, Walters WA, Berg-Lyons D, Lozupone CA Turnbaugh PJ, Knight R. Global patterns of 16S rRNA diversity at a depth of millions of sequences per sample. PNAS. 2011;108(Suppl 1):4516-22. https:// doi.org/10.1073/pnas.1000080107.

23. Bolyen E, Rideout JR, Dillon MR, Bokulich NA, Abnet C, Al-Ghalith GA, Bai Y, et al. QIIME 2: reproducible, interactive, scalable, and extensible microbiome data science. PeerJ Preprints. 2018;6:e27295v1.

24. Callahan BJ, McMurdie PJ, Rosen MJ, Han AW, Johnson AJA, Holmes SP. DADA2: high-resolution sample inference from Illumina amplicon data. Nature Meth. 2016;13(7):581-3. https://doi.org/10.1038/nmeth.3869.

25. Quast C, Pruesse E, Yilmaz P, Gerken J, Schweer T, Yarza P, Peplies J, Glöckner FO. The SILVA ribosomal RNA gene database project: improved data processing and web-based tools. Nucleic Acids Res Spec Publ. 2012; 41(D1):D590-6. https://doi.org/10.1093/nar/gks1219.

26. Segata N, Izard J, Waldron L, Gevers D, Miropolsky L, Garrett WS, Huttenhower C. Metagenomic biomarker discovery and explanation. Genome Biol. 2011;12(6):R60. https://doi.org/10.1186/gb-2011-12-6-r60.

27. Han JE, Tang KF, Tran LH, Lightner DV. Photorhabdus insect-related (Pir) toxin-like genes in a plasmid of Vibrio parahaemolyticus, the causative agent of acute hepatopancreatic necrosis disease (AHPND) of shrimp. Dis Aqua Organ. 2015;113(1):33-40. https://doi.org/10.3354/dao02830.

28. Restrepo L, Bayot B, Betancourt I, Pinzón A. Draft genome sequence of pathogenic bacteria Vibrio parahaemolyticus strain BA94C2, associated with acute hepatopancreatic necrosis disease isolate from South America. Genom Data. 2016;9:143-4. https://doi.org/10.1016/j.gdata.2016.08.008.

29. Grömping, U. Using R and RStudio for Data Management, StatisticalAnalysis and Graphics. Journal of Statistical Software. 2015;68(1):1-7.

30. Koren S, Walenz BP, Berlin K, Miller JR, Bergman NH, Phillippy AM. Canu: scalable and accurate long-read assembly via adaptive $\mathrm{k}$-mer weighting and repeat separation. Genome Res. 2017;27(5):722-36. https://doi.org/10.1101/gr.215087.116.

31. Seemann T. Prokka: rapid prokaryotic genome annotation. Bioinformatics. 2014;30(14):2068-9. https://doi.org/10.1093/bioinformatics/btu153.

32. Aziz RK, Bartels D, Best AA, DeJongh M, Disz T, Edwards RA, Formsma K, Gerdes S, Glass EM, Kubal M, Meyer F, Olsen GJ, Olson R, Osterman AL, Overbeek RA, McNeil LK, Paarmann D, Paczian T, Parrello B, Pusch GD, Reich C, Stevens R, Vassieva O, Vonstein V, Wilke A, Zagnitko O. The RAST Server: rapid annotations using subsystems technology. BMC Genomics. 2008;9(1): 75. https://doi.org/10.1186/1471-2164-9-75.

33. Tatusov RL, Galperin MY, Natale DA, Koonin EV. The COG database: a tool for genome-scale analysis of protein functions and evolution. Nucleic Acids Res Spec Publ. 2000;28(1):33-6. https://doi.org/10.1093/nar/28.1.33.

34. Kanehisa M, Goto S, Kawashima S, Okuno Y, Hattori M. The KEGG resource for deciphering the genome. Nucleic Acids Res Spec Publ. 2004;32(suppl_1): D277-80.

35. Ashburner M, Ball CA, Blake JA, Botstein D, Butler H, Cherry JM, Davis AP, Dolinski K, Dwight SS, Eppig JT, Harris MA, Hill DP, Issel-Tarver L, Kasarskis A, Lewis S, Matese JC, Richardson JE, Ringwald M, Rubin GM, Sherlock G. Gene ontology: tool for the unification of biology. Nat Genet. 2000;25(1):25-9. https://doi.org/10.1038/75556.

36. Clement M, Posada DC, Crandall KA. TCS: a computer program to estimate gene genealogies. Mol Ecol. 2000;9(10):1657-9. https://doi.org/10.1046/j.13 65-294x.2000.01020.x.

37. Tamura K, Stecher G, Peterson D, Filipski A, Kumar S. MEGA6: molecular evolutionary genetics analysis version 6.0. Mol Biol Evol. 2013;30(12):2725-9. https://doi.org/10.1093/molbev/mst197.

38. Darriba D, Taboada GL, Doallo R, Posada D. jModelTest 2: more models, new heuristics and parallel computing. Nat Methods. 2012;9(8):772. https:// doi.org/10.1038/nmeth.2109

39. Darling AC, Mau B, Blattner FR, Perna NT. Mauve: multiple alignment of conserved genomic sequence with rearrangements. Genome Res. 2004; 14(7):1394-403. https://doi.org/10.1101/gr.2289704

40. Bateman A, Coin L, Durbin R, Finn RD, Hollich V, Griffiths-Jones S, et al. The Pfam protein families database. Nucleic Acids Res Spec Publ. 2004; 32(suppl 1):D138-41. https://doi.org/10.1093/nar/gkh121.

41. Soto W, Gutierrez J, Remmenga M, Nishiguchi M. Salinity and temperature effects on physiological responses of Vibrio fischeri from 
diverse ecological niches. Microb Ecol. 2009;57(1):140-50. https://doi. org/10.1007/s00248-008-9412-9.

42. Wang Z, Shi C, Wang H, Wan X, Zhang Q, Song X, Huang J. A novel research on isolation and characterization of Photobacterium damselae subsp. damselae from Pacific white shrimp, Penaeus vannamei, displaying black gill disease cultured in China. J Fish Dis. 2020;43(5):551-9. https://doi. org/10.1111/jfd.13153.

43. Finn RD, Clements J, Eddy SR. HMMER web server: interactive sequence similarity searching. Nucleic Acids Res. 2011;39(suppl2):W29-37. https://doi. org/10.1093/nar/gkr367.

44. Turner JW, Tallman JJ, Macias A, Pinnell LJ, Elledge NC, Azadani DN, et al. Comparative genomic analysis of Vibrio diabolicus and six taxonomic synonyms: a first look at the distribution and diversity of the expanded species. Front Microbiol. 2018;9:1893. https://doi.org/10.3389/ fmicb.2018.01893.

45. Meier-Kolthoff JP, Auch AF, Klenk HP, Göker M. Genome sequence-based species delimitation with confidence intervals and improved distance functions. BMC Bioinformatics. 2013;14(1):60. https://doi.org/10.1186/1471-2105-14-60.

46. Richter M, Rosselló-Móra R. Shifting the genomic gold standard for the prokaryotic species definition. PNAS. 2009;106(45):19126-31. https://doi. org/10.1073/pnas.0906412106.

47. Salomon D, Gonzalez H, Updegraff BL, Orth K. Vibrio parahaemolyticus type $\mathrm{VI}$ secretion system 1 is activated in marine conditions to target bacteria, and is differentially regulated from system 2. PLoS One. 2013;8(4):e61086. https://doi.org/10.1371/journal.pone.0061086.

48. Cornejo-Granados F, Lopez-Zavalan AA, Gallardo-Becerra L, Mendoza-Vargas A, Sánchez F, Vichido R, et al. Microbiome of Pacific Whiteleg shrimp reveals differential bacterial community composition between Wild, Aquacultured and AHPND/EMS outbreak conditions. Sci Rep. 2017;7(1):11783. https://doi. org/10.1038/s41598-017-11805-W.

49. Soonthornchai W, Chaiyapechara S, Klinbunga S, Thongda W, Tangphatsornruang S, Yoocha T, Jarayabhand P, Jiravanichpaisal P. Differentially expressed transcripts in stomach of Penaeus monodon in response to AHPND infection. Dev Comp Immunol. 2016;65:53-63. https:/ doi.org/10.1016/j.dci.2016.06.013.

50. Wang J, Huang Y, Xu K, Zhang X, Sun H, Fan L, Yan M. White spot syndrome virus (WSSV) infection impacts intestinal microbiota composition and function in Litopenaeus vannamei. Fish Shellfish Immun. 2019;84:130-7. https://doi.org/10.1016/j.fsi.2018.09.076.

51. Ding ZF, Cao MJ, Zhu XS, Xu GH, Wang RL. Changes in the gut microbiome of the Chinese mitten crab (Eriocheir sinensis) in response to White spot syndrome virus (WSSV) infection. J Fish Dis. 2017;40(11):1561-71. https://doi. org/10.1111/jfd.12624.

52. Neulinger SC, Gärtner A, Järnegren J, Ludvigsen M, Lochte K, Dullo WC. Tissue-associated "Candidatus Mycoplasma corallicola" and filamentous bacteria on the cold-water coral Lophelia pertusa (Scleractinia). Appl Environ Microbiol. 2009;75(5):1437-44. https://doi. org/10.1128/AEM.01781-08.

53. Holm JB, Heidelberg KB. Microbiomes of Muricea californica and M. fruticosa: comparative analyses of two co-occurring eastern Pacific octocorals. Front Microbiol. 2016:7:917.

54. Weiland-Bräuer N, Neulinger SC, Pinnow N, Künzel S, Baines JF, Schmitz RA. Composition of bacterial communities associated with Aurelia aurita changes with compartment, life stage, and population. Appl Environ Microbiol. 2015;81(17):6038-52. https://doi.org/10.1128/AEM.01601-15.

55. Rua CP, Trindade-Silva AE, Appolinario LR, Venas TM, Garcia GD, Carvalho LS, et al. Diversity and antimicrobial potential of culturable heterotrophic bacteria associated with the endemic marine sponge Arenosclera brasiliensis. PeerJ. 2014;2:e419. https://doi.org/10.7717/peerj.419.

56. Yu W, Wu JH, Zhang J, Yang W, Chen J, Xiong J. A meta-analysis reveals universal gut bacterial signatures for diagnosing the incidence of shrimp disease. FEMS Microb Ecol. 2018;94(10):147.

57. Woo HA, Chae HZ, Hwang SC, Yang KS, Kang SW, Kim K, Rhee S. Reversing the inactivation of peroxiredoxins caused by cysteine sulfinic acid formation. Sci. 2003;300(5619):653-6. https://doi.org/10.1126/ science. 1080273.

58. Hasan NA, Grim CJ, Lipp EK, Rivera IN, Chun J, Haley BJ, et al. Deep-sea hydrothermal vent bacteria related to human pathogenic Vibrio species. PNAS. 2015;112(21):E2813-9. https://doi.org/10.1073/pnas.1503928112.

59. Yévenes M, Quiroz M, Maruyama F, Jorquera M, Gajardo G. Vibrio sp. ArtGut$\mathrm{Cl}$, a polyhydroxybutyrate producer isolated from the gut of the aquaculture live diet Artemia (Crustacea). Electron J Biotechnol. 2021;49:228. https://doi.org/10.1016/j.ejbt.2020.10.003.

60. Galán JE, Lara-Tejero M, Marlovits TC, Wagner S. Bacterial type III secretion systems: specialized nanomachines for protein delivery into target cells. Annu Rev Microbiol. 2014;68(1):415-38. https://doi.org/10.1146/annurevmicro-092412-155725.

61. Wang R, Zhong Y, Gu X, Yuan J, Saeed AF, Wang S. The pathogenesis, detection, and prevention of Vibrio parahaemolyticus. Front Microbiol. 2015;6:144.

62. Carpenter MR, Kalburge SS, Borowski JD, Peters MC, Colwell RR, Boyd EF. CRISPR-Cas and contact-dependent secretion systems present on excisable pathogenicity islands with conserved recombination modules. J Bacteriol . 2017 Apr 25;199(10):e00842-16. https://doi.org/10.1128/JB.00842-16.

63. Salomon D, Kinch LN, Trudgian DC, Guo X, Klimko JA, Grishin NV, Mirzaei H, Orth K. Marker for type VI secretion system effectors. PNAS. 2014;111(25): 9271-6. https://doi.org/10.1073/pnas.1406110111.

64. Weber B, Hasic M, Chen C, Wai SN, Milton DL. Type VI secretion modulates quorum sensing and stress response in Vibrio anguillarum. Environ Microbiol. 2009;11(12):3018-28. https://doi.org/10.1111/j.1462-292 0.2009.02005.x.

\section{Publisher's Note}

Springer Nature remains neutral with regard to jurisdictional claims in published maps and institutional affiliations.
Ready to submit your research? Choose BMC and benefit from:

- fast, convenient online submission

- thorough peer review by experienced researchers in your field

- rapid publication on acceptance

- support for research data, including large and complex data types

- gold Open Access which fosters wider collaboration and increased citations

- maximum visibility for your research: over $100 \mathrm{M}$ website views per year

At $\mathrm{BMC}$, research is always in progress.

Learn more biomedcentral.com/submissions 